\title{
Functionalized Polyelectrolytes for Bioengineered Interfaces and Biosensing Applications
}

\author{
Jacopo Movillia (D) \\ Jurriaan Huskens*a (i) \\ a Molecular NanoFabrication group, MESA+ Institute for Nanotechnology, Department \\ of Science and Technology, University of Twente, P.O. Box 217, 7500 AE, Enschede, \\ The Netherlands \\ j.huskens@utwente.nl
}

Received: 2.12.2019

Accepted after revision: 31.01 .2020

DOI: 10.1055/s-0040-1708494; Art ID: 190024

License terms: CCO

(c) 2019. The Author(s). This is an open access article published by Thieme under the terms of the Creative Commons Attribution-NonDerivative-NonCommercial-License, permitting copying and reproduction so long as the original work is given appropriate credit. Contents may not be used for commercial purposes, or adapted, remixed, transformed or built upon. (https://creativecommons.org/licenses/by-nc-nd/4.0/).

Abstract The possibility of tuning the chemical moieties and their density plays a fundamental role in targeting surface-confined molecular structures and their functionalities at macro and nanoscale levels. Such interfacial control is crucial for engineered coating formation and biorecognition purposes, where the type and density of ligands/receptors at the surface affect the overall binding affinities and the device performance. Together with the well-established selfassembled monolayers, a surface modification approach based on polyelectrolytes (PEs) has gained importance to provide desired characteristics at the substrate interface. This review presents the innovations of functional PEs, modified in a preceding synthetic step, and their wide applicability in functional (a)biotic substrates. Examples of $2 \mathrm{D}$ and $3 \mathrm{D}$ architectures made by modified PEs are reviewed in relation with the reactive groups grafted to the PE backbones. The main focus lies on the strategy to use modified PEs to form bioengineered coatings for orthogonally anchoring biological entities, manufacturing biocidal/antifouling films, and their combinations in functional biosensing applications.

Key words polyelectrolytes, self-assembly, surface modification, bioengineered interfaces, biosensing, adsorption

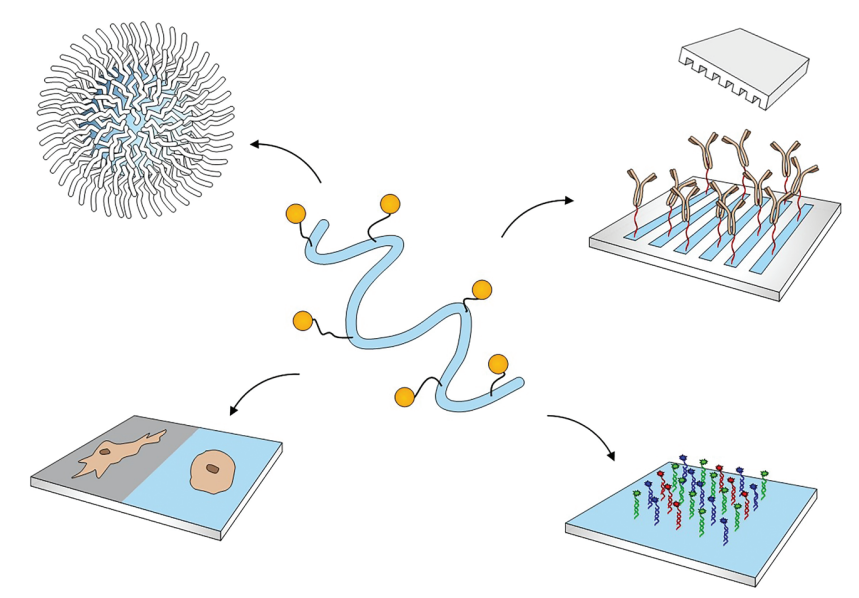

Introduction

The process of engineering different types of interfaces while controlling their physicochemical properties is a multidisciplinary challenge with boundless applications, ranging from the macro to the nanoscale. ${ }^{1,2}$ Coating surfaces with functional moieties is a widely used approach in mechanical industries and tribology to reduce friction and increase lubrication (Figure 1a), ${ }^{2}$ in manufacturing to prevent rust and degradation, ${ }^{3}$ as well as in food packaging to prevent bacteria growth. ${ }^{4}$ Similar properties are frequently required in nano(bio)technology. Homogeneous electrodeposition of metals for photovoltaic cells, ${ }^{5}$ passivation of implantable devices to avoid inflammatory responses, ${ }^{6}$ antifouling coatings for biosensing/point-ofcare devices (Figure 1b), ${ }^{7,8}$ and hydrogel layers to increase the hydration of biochips ${ }^{9}$ are a few examples of the plethora of features addressed by functional interfaces.

The establishment and regulation of desired characteristics onto virtually any substrate has been the ultimate aim for surface chemistry. At the nanoscale level, especially in materials science and biomedical applications, the study and development of tunable and universal surface engineering methods have become of utmost importance. Love et al. described this as: "[unlike bulk materials] a high percentage of the constituent atoms are located at the surface. Therefore, the properties of nanostructured materials depend to a much greater extent on their surface and interfacial environment than do bulk materials." ${ }^{10}$ However, in concert with the characteristics of the formed layer, the introduction of secondary functionalities at the interface in a controlled and (bio)orthogonal fashion has been a crucial point for developing efficient surface modification strategies. ${ }^{11}$ For instance, while designing 
Biographical Sketch
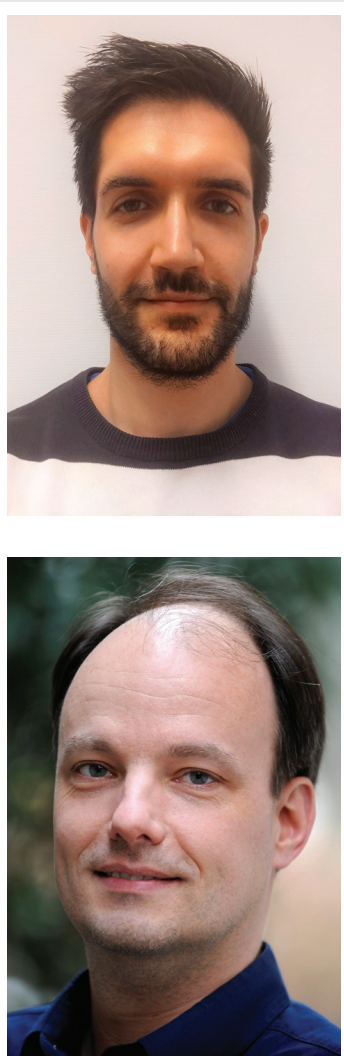

Jurriaan Huskens (1968) obtained his PhD (1994) at the Delft University of Technology with Herman van Bekkum. After postdoctoral stays with Dean Sherry and Manfred Reetz, he became assistant professor with David Reinhoudt at the University of
During his Master's at the University of Padova (2015), he worked in the Supramolecular Chemistry and Functional Nanoparticles group of Prof. Fabrizio Mancin. In September 2015, he joined the Molecular Nanofabrication group at the University of Twente under the supervision of Prof. Jurriaan Huskens. His project, which is part of the European project Ultraplacad for early cancer diagnosis, focuses on the applicability of engineered polyelectrolytes at surfaces for biorecognition and biosensing applications.
Twente in 1998, and full professor in 2005. He received the Unilever Research Award (1990), a Marie Curie fellowship (1997), the Gold Medal 2007 of the Royal Netherlands Chemical Society, and a fellowship from the Institute of Advanced Study, Durham
University, UK (2019). Present research interests encompass supramolecular chemistry at interfaces, supramolecular materials, multivalency, nanofabrication, and solar fuels. He is a (co) author of about 400 refereed research papers and five patents. scaffolds for drug/gene delivery or imaging applications, the layout of the outermost layer needs to be carefully taken into account since it will affect the pharmacokinetic profile and the interactions with the biological system. ${ }^{12,13}$ Typically, such scaffolds require a camouflaging shield to reduce immunogenicity and rapid clearance, which is usually accomplished by the addition of zwitterionic groups, ${ }^{14,15}$ poly(ethylene glycol) (PEG), ${ }^{16,17}$ or polyoxazo-

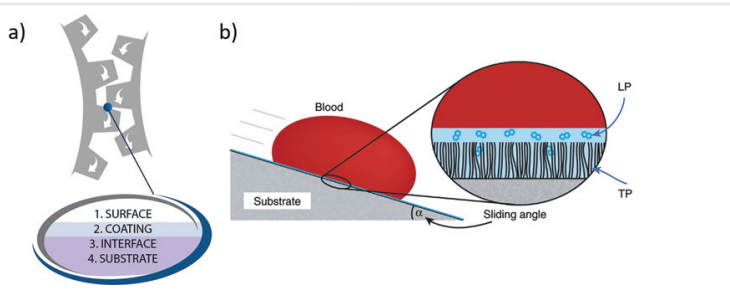

Figure 1 Examples of engineered coatings. (a) Illustration showing tribological properties in sensitive parts of a general gearwheel connection. (b) Schematic representation of a blood drop sliding on a repellent surface formed by binding a flexible tethered perfluorocarbon layer and then coating it with a mobile layer of perfluorodecalin. Reprinted with permission from Ref. 7. Copyright 2014 Springer Nature. line chains. ${ }^{18,19}$ Moreover, specific receptors for active targeting can also be included. ${ }^{20,21}$

The same antifouling moieties have been exploited in biosensing and point-of-care devices to minimize nonspecific interactions at the interface of the transducer device, $^{22-25}$ where the translation of a biological binding event into an electrical/optical signal occurs. Yet, the integration of the receptor at the transducing surface remains the pivotal step in order to achieve excellent selectivity and sensitivity. ${ }^{26,27}$ Not only the density, but also the conformation and orientation of the receptor can affect the final outcome of the sensing device. ${ }^{28,29}$ For example, the denaturation of antibody and protein probes, ${ }^{30,31}$ as well as the electrostatic and steric hindrance of nucleotide receptors, ${ }^{32,33}$ can result in lower signals. Therefore, the controlled thin film formation and binding of (bio)molecules at the interface is a key aspect toward effective modification strategies for biorecognition and bioresponsive surfaces. In addition, the type of transducing substrate deserves particular focus for its crucial role to achieve the highest possible sensitivity. Depending on the variation of the physical property affected by the bimolecular recognition event (e.g., refractive index, electrical resistance, fluorescence/light intensity, and mass change), different materials are selected for the transducing substrate. 
Consequently, the surface functionalization method is often dictated by the material type of the transducer. ${ }^{34,35}$

Surface modification strategies have been prevalently based on chemisorption methods. ${ }^{36}$ Due to the necessity of forming strong, surface-specific bonds, as well as to fulfill the aforementioned requirements for the transducing process, biosensing substrates have been made of metals [mostly gold (Au) and silver $(\mathrm{Ag})],{ }^{37-40}$ silicon-based materials (mainly $\left.\mathrm{SiO}_{2}\right)^{41-43}$ graphene, ${ }^{44,45}$ and metal oxides including $\mathrm{TiO}_{2},{ }^{46,47} \mathrm{Al}_{2} \mathrm{O}_{3},{ }^{48,49}$ and indium tin oxide (ITO). ${ }^{50,51}$ In the 1980s, the first elegant solution for controlling film formation and molecule distribution at a surface was created through self-assembled monolayers (SAMs) based on thiol-Au ${ }^{52,53}$ and thiol- $\mathrm{Ag}^{54,55}$ interactions, as well as silanization protocols on $\mathrm{SiO}_{2}$ substrates. ${ }^{56,57}$ Since then, engineered SAM approaches have been exploited to form responsive biosensor interfaces. Highly packed, mixed SAMs assure control over both accessibility and molecular orientation for selective biomolecule immobilization, ${ }^{58,59}$ in concert with antifouling properties required from the film. ${ }^{60}$ In addition, the easy monolayer formation and the tunable probe density provide valuable features, which have contributed to making the SAM approach the most used surface modification strategy. ${ }^{61} \mathrm{~A}$ comprehensive variety of functional SAMs has been documented for anchoring or detecting target DNA molecules, ${ }^{62,63}$ proteins, ${ }^{64,65}$ entire cells, ${ }^{66,67}$ peptides, ${ }^{68,69}$ aptamers, ${ }^{70,71}$ and other biologically relevant molecules for biosensing purposes. A full coverage of SAM approaches at transducer interfaces and their tunable properties and multifunctional architectures for biosensing purposes is beyond the scope of this review.

Polyelectrolyte (PE) physisorption has emerged as a general and cost-effective surface functionalization method to homogeneously form chemical architectures on different substrates. ${ }^{72,73}$ PEs belong to a subfamily of polymers, where the monomers (or subunits) carry an electrolyte functionality, typically a charged organic moiety. Therefore, various (bio) molecules can be identified as PEs and multiple classifications can be made (i.e., based on synthesis, biological/artificial origin, type of monomers, resulting charges, etc.). These classifications and the synthesis/production of PEs will not be discussed in this review. Yet, PEs are mainly categorized into two groups: strong PEs, when the (de)protonatable groups dissociate completely in solution across a wide $\mathrm{pH}$ range, and weak PEs if deprotonation only occurs to a certain extent. Thus, strong PEs have permanent charges, while weak PEs are partially charged at intermediate $\mathrm{pH}(\sim 2-10)$ and the charged fraction can be tuned by varying the $\mathrm{pH}$ or ionic strength.

PEs represent a significant innovation for the creation of functional biorecognition interfaces. Despite their weak (and mainly electrostatic) bond formation, in comparison to the stronger $\mathrm{Au}$-thiol SAM formation, the multivalent nature of the PE backbones ensures almost irreversible adhesion of aqueous solution onto substrates with the opposite charge. ${ }^{36,74,75}$ Moreover, the process does not require organic solvents. The possibility of using aqueous solutions and biological buffers, with defined $\mathrm{pH}$, has the double benefit of controlling the amount of ionized groups at the PE side chains and assuring biocompatibility for the immobilization of biological macromolecules. ${ }^{76-78}$ Modified PEs can be adsorbed virtually on any type of substrate, such as metals, metal oxides, and polymeric materials, ${ }^{79,80}$ resulting in a general and customizable surface functionalization method for engineered coatings and for a broad range of biosensing applications. ${ }^{81}$ However, the introduction of specific functionalities on PE-based films needs to be performed orthogonally and in a reliable way.

Biorecognition interfaces require selectivity and sensitivity. Only the target analyte should be detected, while at the same time giving the highest possible detectable change upon binding. The design of the biorecognition surface is critical to reach these standards. In particular, the type, orientation, and density of active molecules have to be tailored in concert with the surrounding environment. ${ }^{82-84}$ Therefore, the controlled display of such entities at the interface is essential to avoid possible interfering complexations or the occurrence of steric and electrostatic repulsion toward the analyte, which hamper the performance of the sensor. An approach based on introduction of functional moieties after the PE film formation in a covalent or noncovalent way (postfunctionalized PEs) requires further film characterization. In order to quantify the receptor density and the hybridization efficiency (for nucleotidebased sensors) after the sample preparation, additional, and sometimes invasive, surface-analytical techniques are needed. ${ }^{85-88}$ However, we have recently showed that the covalent binding of such moieties in a synthetic step preceding the PE assembly process can assure control of the biomolecule density that is displayed at the interface. ${ }^{89}$ Quantification of the grafted groups can be easily achieved by solution analysis techniques, after which the PE surface self-assembly is performed and further (macro)molecules can be anchored subsequently. ${ }^{90,91}$ Through the control of the molecule immobilization and their density, high reproducibility and consistency can be achieved, which is of utmost importance for biosensing applications.

In this review, an overview is presented of surface modification strategies based on modified PEs, with an emphasis on PEs that are functionalized in a synthetic step preceding the surface assembly. In our view, modified PEs provide a generic way of functionalizing a wide range of substrate materials and devices, thus providing a platform technology for a wide range of applications. A particular focus is put on the use of PEs for functional engineered interfaces and biosensing purposes. The general applicability of the PE method is described together with the variety of materials that can be used, including the features to promote the molecular self-assembly. Different layer 
architectures are described together with the type of substrate, including micro- and nanostructured interfaces, hydrogels, patterning techniques and nanoparticles (NPs). Specifically, in the first part, combinations of multiple grafted functionalities are presented, while the second part aims at providing an overview of various functional engineered coatings and biosensing applications. Block co-PEs, post-functionalized PEs (functionalized after their surface assembly), conjugated PEs, PE brushes, and PE complexes for drug delivery are not included in this overview.

\section{Polyelectrolyte Adsorption}

The intimate relationship between the deposited molecules and the substrate is a key factor in determining the characteristics of the functional interface. Several papers have discerned, both experimentally and theoretically, the adsorption behavior of strong and weak PEs on a variety of substrates. ${ }^{92-95}$ The densities of the charges present on both the surface and the PE backbone, together with the salt concentration, were found to be the main driving force for the adsorption process. ${ }^{96,97}$ Hoogeveen et al. investigated the adsorption of strong, quaternized polyvinylpyridine $\left(\mathrm{PVP}^{+}\right)$on untreated $\mathrm{TiO}_{2}$ and $\mathrm{SiO}_{2}$ and that of quaternized poly(dimethylaminoethyl methacrylate) $\left(\mathrm{AMA}^{+}\right)$and its parent, weak AMA, on $\mathrm{TiO}_{2} \cdot{ }^{97}$ With this combination of substrates and PEs, the effect of the strong/weak nature (i.e., the persistence of charges at the PE backbones after dissolution) was studied in relation to the surface charges and ionic strength. The strong PEs PVP ${ }^{+}$ and $\mathrm{AMA}^{+}$showed fast adsorption kinetics on $\mathrm{TiO}_{2}$ with almost complete coverage within $5 \mathrm{~min}$. The absence of adsorption for the strong PEs at high ionic strength confirmed the predominant electrostatic effect, as shown for other systems. ${ }^{98,99}$ However, while the adsorption of all three PEs was electrostatic on $\mathrm{TiO}_{2}, \mathrm{PVP}^{+}$still presented some adsorption at high ionic strength for $\mathrm{SiO}_{2}$, suggesting an additional contribution of nonelectrostatic forces, due to the pyridinyl ring. The effect of surface charge was investigated by varying the $\mathrm{pH}$ during the adsorption of $\mathrm{PVP}^{+}$and $\mathrm{AMA}^{+}$on $\mathrm{TiO}_{2}$. Because of the isoelectric point of $\mathrm{TiO}_{2}$ (around $\mathrm{pH} 4$ ), the adsorbed amount of $\mathrm{AMA}^{+}$ increased linearly with the $\mathrm{pH}$ (Figure 2a, triangles), suggesting a strong dependence on the surface charge density. On the other hand, deposition of AMA was affected strongly by the $\mathrm{pH}$, due to its weak nature (Figure 2a, open and filled circles for different ionic strengths). By increasing the $\mathrm{pH}$ from 3 to 9, more PE molecules (with fewer charges per $\mathrm{PE}$ ) were needed to compensate the surface charge, resulting in more material deposited. The continued depletion of positive charges on AMA started to hinder the adsorption above $\mathrm{pH}$ 9. These combined effects created a binding maximum that is typical of the combination of a substrate and a weak PE. A similar adsorption profile has been observed by Böhmer et al. for poly(acrylic acid) (PAA) on polystyrene (PS) and confirmed with a model, demonstrating at the same time the main role of $\mathrm{pH}$ in controlling the degree of ionization for weak PEs, as well as the independence from the substrate. ${ }^{100,101}$

The $\mathrm{pH}$ dependence of the surface charges for a specific substrate can be mitigated by working at relatively high salt concentrations ${ }^{74}$ or by introducing charges on top of the surface by means of activation or prefunctionalization methods (such as the use of a charged SAM). Lappan and coworkers showed that oxygen plasma treatment creates stable, negative zeta potential values for poly

\section{a)}

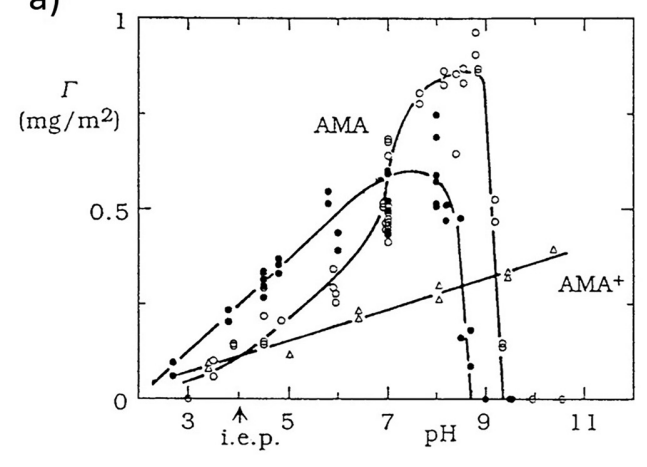

b)

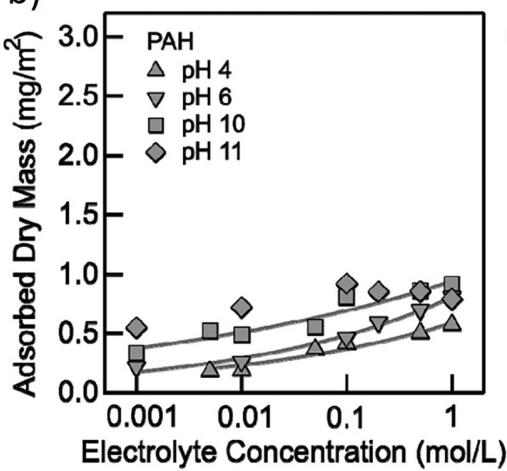

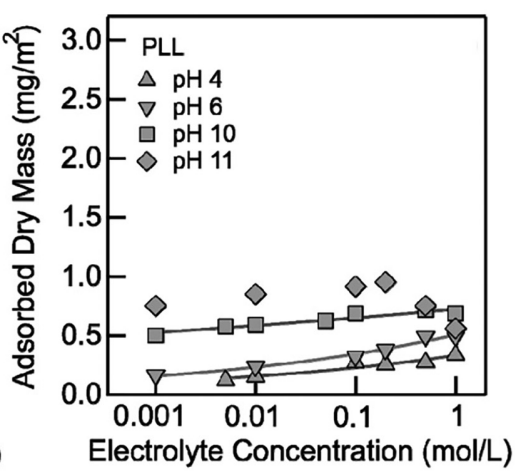

Figure 2 Adsorbed dry mass of PE versus pH determined by reflectometry measurements for (a) strong $\mathrm{AMA}^{+}$(triangles) and weak AMA (open circles) at ionic strength $0.005 \mathrm{M}$ and weak AMA at ionic strength $0.1 \mathrm{M}$ (filled circle) on inactivated $\mathrm{TiO}_{2}$ substrates (PE concentration: $0.01 \mathrm{mg} / \mathrm{mL}$ ). Reprinted with permission from Ref. 97. Copyright 1996 Elsevier. (b) Dry mass adsorbed versus electrolyte concentration in solution for weak $\mathrm{PAH}$ and $\mathrm{PLL}$ on $\mathrm{SiO}_{2}$ pretreated with oxidative treatment by UV-ozone (PE concentration: $0.005 \mathrm{mg} / \mathrm{mL}$ ). Adapted with permission from Ref. 76 . Copyright 2012 American Chemical Society. 
(tetrafluoroethylene) (PTFE) substrates in a wide range of $\mathrm{pH}^{102}$ Strong poly(diallyldimethylammonium chloride) (PDADMAC) could then been adsorbed electrostatically. Porus and collaborators investigated the structure of three adsorbed monolayers of poly(styrene sulfonate) (PSS), poly (allylamine hydrochloride) (PAH), and poly-L-lysine (PLL) by means of reflectometry and quartz crystal microbalance equipped with dissipation (QCM-D). ${ }^{76}$ Interestingly, the amount of PE adsorbed at various $\mathrm{pH}$ values for the weak PAH $\left(\mathrm{p} K_{\mathrm{a}}=\sim 9\right)^{103}$ and PLL $\left(\mathrm{p} K_{\mathrm{a}}=\sim 10\right)^{104}$ on $\mathrm{SiO}_{2}$ (preactivated by UV-ozone) did not show the "bell" shape found by Hoogeveen et al. for AMA (Figure 2a, open and filled circles) and Böhmer et al. for the polyanion PAA, ${ }^{101}$ but kept on increasing at higher $\mathrm{pH}$ (Figure 2b). PAH and PLL adsorption profiles showed a relationship more similar to strong PEs such as $\mathrm{AMA}^{+}$(Figure 2a, triangles) or PDADMAC. ${ }^{94}$ This was ascribed to the density of charges present at the surface, previously formed by oxidative treatment. Choi et al. compared the adsorption of PLL expressing strong and weak behaviors when varying the $\mathrm{pH}$ from 2 to 12 on plasma-activated $\mathrm{SiO}_{2}$ and Au substrates, precoated with either amino or carboxylic acid-terminated SAMs. ${ }^{105}$ The highest adsorbed mass was observed for the $\mathrm{COOH}$-terminated SAM, while the smoothest and most uniform PE monolayer was detected by atomic force microscopy (AFM) on silica, suggesting that the amount and homogeneous distribution of charges on the surface results in highly homogeneous, thin PE monolayers.

The universality of the PE approach has been supported by studies on many other combinations of PEs and activated substrates, such as polyethylenimine (PEI) on polyethylene, ${ }^{106}$ ITO, ${ }^{107}$ poly(dimethylsiloxane) (PDMS), ${ }^{108}$ cyclic olefin polymer (COP), and glass ${ }^{109}$; PLL on $\mathrm{Au}^{110}$ and PDMS $^{111}$; and PAH on PDMS ${ }^{112}$ and PTFE, ${ }^{113}$ where the materials have been previously treated with plasma cleaning or UV-ozone. Even harsher procedures, such as acidic or basic piranha solutions, were used, for example, to anchor $\mathrm{PEI}^{114}$ or $\mathrm{PAA}^{115}$ on silicon, $\mathrm{PVP}^{+}$on $\mathrm{Au},{ }^{116}$ and $\mathrm{PAH}$ on glass. ${ }^{117}$

PEs can also adsorb on surfaces that naturally present a certain density of charges such as $\mathrm{SiO}_{2},{ }^{118} \mathrm{TiO}_{2},{ }^{119}$ stainless steel $^{120}$ as well as Fe and $\mathrm{Al}$ oxide/hydroxides, ${ }^{121}$ even without activation methods or the preformation of a charged SAM on the substrate. As explained by Szilagyi et al. in their review, not only the predominant electrostatic interactions but also additional van der Waals, hydrophobic, and depletion forces determine the layer formation. ${ }^{122}$ They described the adsorption on both planar and curved substrates, with a clear focus on the layer morphology and saturation. They observed that film saturation points, at which PEs did not adsorb anymore, were not caused by the surface charges, but rather by the local environment of the adsorbing PE chains. Apparently, at appropriate concentrations of PE and salt, the PE will eventually occupy an empty spot, forming uniform films regardless the total density of charges at the surface. Consequently, NPs and morphologically complex nanostructures could be coated homogeneously with PEs. Some examples include NPs made of $\mathrm{Au},{ }^{123} \mathrm{Al},{ }^{124} \mathrm{ZnO},{ }^{125}$ and charged PS beads, ${ }^{126}$ as well as nanoporous anodized $\mathrm{Al}$ oxide, ${ }^{127} \mathrm{Au}$ nanoflowers, ${ }^{128}$ nanodendrites, ${ }^{129}$ and even hydrogels. ${ }^{130}$

The PE surface functionalization strategy has established new forms of control for molecular self-assembly with direct consequences for the substrate applicability. In the rest of this section, the introduction of functional moieties at the PE backbone in a preceding synthetic step and the quantification of the grafting density and its role in tuning the density of groups upon surface adsorption will be presented. A particular focus is put on the use of specific chemical moieties and their combinations to create uniform structured 2D, 3D, and patterned functional interfaces.

\section{Monolayers of Modified PEs}

As explained above, grafting functional molecules to PEs during a synthetic step preceding the adsorption can offer an appealing solution for the design of biosensing surfaces. Appropriate spectroscopic techniques, such as NMR, are then required for the quantification of the appended groups, after which the PE molecular self-assembly on the desired substrate can take place. Their assembly then leads to the controlled display of chemical moieties on the film.

One of the first examples of biosensing with a modified $\mathrm{PE}$, in order to form an electrochemical sensor for glucose and lactate, was presented by the group of Battaglini. ${ }^{131}$ Following the pioneering work of Forster and Vos, ${ }^{132}$ Battaglini and coworkers coupled a redox osmium complex to $\mathrm{PAH}$ and applied it on a glassy carbon electrode. By reacting $\left(\mathrm{Os}(\mathrm{bpy})_{2} \mathrm{Cl}(\mathrm{pyCHO})\right) \mathrm{Cl}$ to the amine groups of $\mathrm{PAH}$, they formed an Os-PAH derivative, which was characterized by spectrophotometry, giving a ratio of amino groups:Os of $8: 1$. The tunable amount of active Os species was essential to form an electrically stable interface. Yang et al. developed a biocompatible redox polymer by coupling ferrocenecarboxaldehyde to chitosan. ${ }^{133}$ The degree of functionalization was quantified by ${ }^{1} \mathrm{H}-\mathrm{NMR}$ and FT-IR. Chitosan with appended ferrocene ( $\mathrm{Fc}$ ) groups (Figure 3a) was employed to form an enzyme-based glucose biosensor. Here, the Fc moieties acted as redox mediators to provide a more efficient electron transfer in the electrochemical oxidation of the analyte. The intrinsic sensitivity was amplified by increasing the fraction of Fc during the synthetic step. In this and other successful examples based on the same modified PEs, ${ }^{134,135}$ the enzymes were physisorbed, while subsequent assembly of the PE provided a stabilizing environment.

Another way of controlling the receptor density was achieved by tailoring the number of receptors grafted to the 


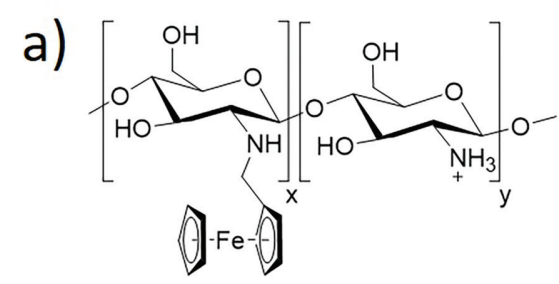

C)

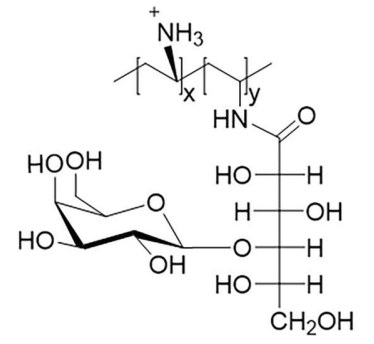

b)

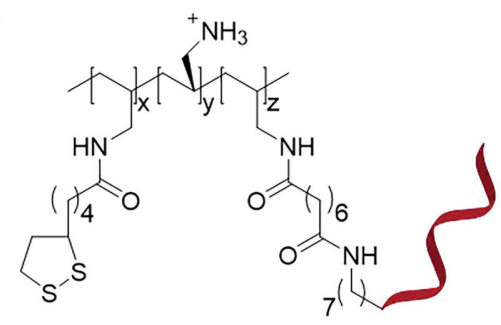

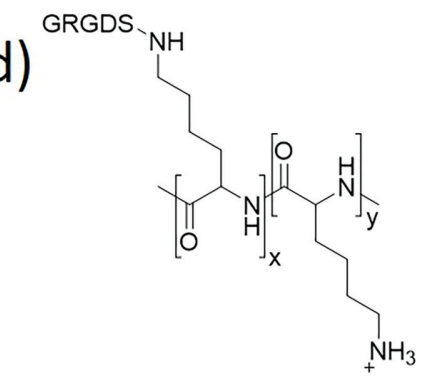

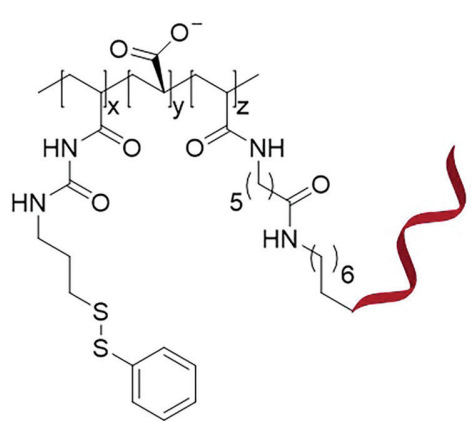

e)

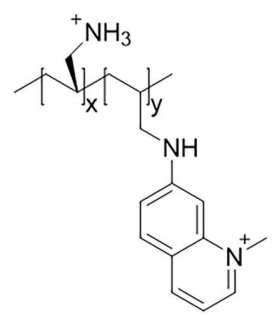

Figure 3 (a) Chitosan-Fc polyelectrolyte used to stabilize the enzyme glucose oxidase and to amplify the electrochemical response. ${ }^{133}$ (b) PAH and PAA functionalized with anchoring disulfide units and a ssDNA probe for direct detection after surface assembly. ${ }^{136}$ (c) PVAm-Gal for galactose-specific lectin recognition. ${ }^{138}$ (d) PLL-GRGDS (containing the RGD sequence) to monitor the receptor-mediated cell interaction at the surface. ${ }^{139}$ (e) Fluorescently labeled PAH for fingerprint staining. ${ }^{141}$

PE backbone in a preceding synthetic step, which directly influences the accessibility of the analyte toward the interface. This approach allowed performing the measurement right after the assembly and it improved the reproducibility of the assay. Taira and coworkers grafted single-stranded DNA (ssDNA) to either PAH or PAA (Figure $3 \mathrm{~b}$ ) with a maximum grafting percentage of 0.5. ${ }^{136,137}$ In order to assist the PE anchoring onto Au, two different disulfide units were attached to the backbones. Mokhtari et al. varied the amount of lactobionic acid, which contains the galactose (Gal) moiety, during the anchoring step to the amino group of poly(vinyl amine) $(\mathrm{PVAm}){ }^{138}$ A small library of PVAm-Gal polymers (Figure 3c) with grafting densities ranging from 6 to 23\% were synthesized and characterized by both ${ }^{1} \mathrm{H}-\mathrm{NMR}$ spectroscopy and conductometric titrations, aimed at selectively detecting galactose-binding lectins. Another example of the controlled display of bioactive molecules was shown by Quirk et al. for the formation of tissue-like cell binding on poly(lactic acid) films. ${ }^{139}$ By varying the density of Arg-Gly-Asp oligopeptides (known as the RGD sequence, which reportedly binds to cell integrin receptors promoting growth and differentiation $)^{140}$ on the PLL backbone (Figure 3d), the receptor-mediated response of cells was tuned. Modified PEs have been used to control the deposition of chromophores on surfaces. Van der Mee and coworkers grafted PAH with 7-amino quinolinium
(Figure 3e) to efficiently stain fingerprints. ${ }^{141}$ However, nonspecific interactions were reported for all the previous examples.

To promote only the specific interactions to occur at the biorecognition interface, antifouling moieties are often required. It is of utmost importance that the surface density of probes or active biomolecules is controlled together with the antifouling behavior of the film to maximize the sensitivity and selectivity. The capability of minimizing nonspecific interactions at the interface without affecting the analyte detection has presented a major challenge for biosensors and bioresponsive coatings. ${ }^{142,143}$ Already in the 1990s, PEI was used to produce nonfouling films by the addition of PEG or ethyl hydroxyethyl cellulose (EHEC). These PEI films were shown to more efficiently repel proteins than grafting PEG chains postassembly. ${ }^{144}$ In the same years, Sawhney and Hubbel showed the first example of PEG grafted PLL (PLL-PEG) to improve the biocompatibility of alginate microcapsules and reduce nonspecific interactions. ${ }^{145}$ With an average of 1 PEG chain every 10 Lys units (quantified by ${ }^{1} \mathrm{H}-\mathrm{NMR}$ ), they showed reduced adsorption of bovine serum albumin (BSA) and fibrinogen (Fg) on a glass slip coated with PLL-PEG when compared with unmodified alginate and unfunctionalized PLL. Thereafter, the groups of Hubbell, Vörös, Spencer, and Textor published several studies on protein-resistant PLL-PEG interfaces, investigating the stability under different 
conditions and on multiple substrates, varying the grafted mole fractions and molecular weights of both components. ${ }^{146-150}$ All in all, the use of PEG-PLL established the most used and cost-effective way of passivating surfaces to uniformly display antifouling behavior.

Other moieties have been appended to the PLL backbone to increase the protein resistance of the interface after selfassembly. For example, Pidhatika et al. and Konradi et al. introduced poly(2-methyl-2-oxazoline) (PMOXA) as side chains to a PLL backbone as a new nonfouling coating material. ${ }^{151,152}$ Morgese and coworkers compared the efficacy of coated $\mathrm{SiO}_{2}$ surfaces in resisting nonspecific protein adsorption using four different moieties grafted to the PLL backbone. ${ }^{153}$ Next to the well-known PEG, hydrophilic variants of poly(2-alkyl-2-oxazoline)s (PAOXAs), such as PMOXA, poly(2-ethyl-2-oxazoline) (PEOXA) and poly(2-methyl-2-ozazine) (PMOZI), were reacted with PLL to functionalize approximately $30 \%$ of the lysine monomers (Figure 4a). The combination of flexibility and hydration of the chains served as an explanation for the excellent resistance to protein adsorption from human and fetal bovine serum (Figure 4b). A biomimetic alternative to PEG and polyoxazoline was recently reported based on the pioneering work of Löfås on dextran-coated surfaces. ${ }^{154}$ Holland and coworkers grafted dextran oligomers to PVAm together with hexanoyl or lauroyl units to mimic the external region of a cell membrane, known as the glycocalyx, on graphite surfaces. ${ }^{155}$ The group of Spencer reported a PLL-dextran copolymer to reduce the protein adsorption also on silica-titania substrates. ${ }^{156}$

Besides nonfouling properties, it is necessary to avoid any repulsive effect on the desired target analyte. ${ }^{157,158}$ Therefore, a biorecognition interface requires the presence and the availability of a specific receptor (or a bioactive molecule) to perform the binding process. An elegant strategy for the formation of such a layer is the use of a spacer to assure the accessibility and directionality of the receptor (e.g., RGD peptide, nitrilotriacetic acid) or the reactive linker (e.g., biotin, maleimide) after the PE selfassembly at the interface. ${ }^{159}$ In the former case, the measurement can be performed right after the PE selfassembly at the interface, ${ }^{158,160}$ while in the latter case, additional step(s) are required to anchor the receptor [e.g., streptavidin (SAv)-biotinylated probe, thiolated probe]. ${ }^{78,161,162}$ Although the first example is the more straightforward, the second one is preferred for bulky probes or charged macromolecules in order to avoid PEprobe complexes or co-adsorption at the surface. Such processes can result in a decrease of the receptor activity as well as precluding uniform film formation. For both examples, the density of grafted molecules (of receptor/reactive linker and antifouling groups) is known before the PE adsorption. The distribution of bioactive entities at the interface is directly related to the density of the previously grafted end-functionalized spacer. ${ }^{163,164}$ Therefore, the proper design of modified PEs with appended antifouling spacers, which are end-functionalized with a receptor or orthogonal reactive linker, results in an assembled film having nonfouling and functional moieties positioned on top of the film. ${ }^{158}$ The nature of the interactions between the surface and the individual anchoring units of PEs is the reason for such a bilayered functional group/PE structure. ${ }^{36}$

Reimhult and Höök discussed that the key aspect of (modified) PEs as coating materials is the uniform layer formation owing to the reversibility of the charged monomers physisorbed to the surface. ${ }^{36}$ This property allows multiple rearrangements at the interface to create more packed, homogeneous coatings and fewer surface a)

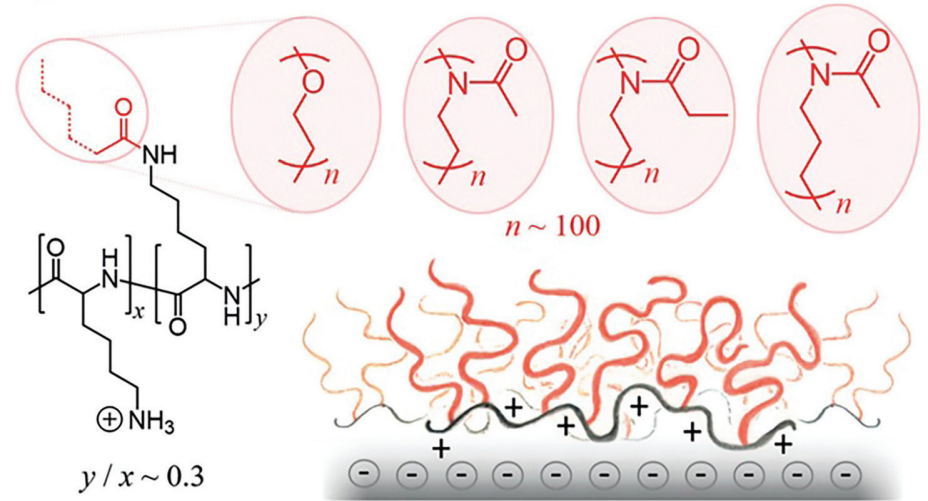

b)

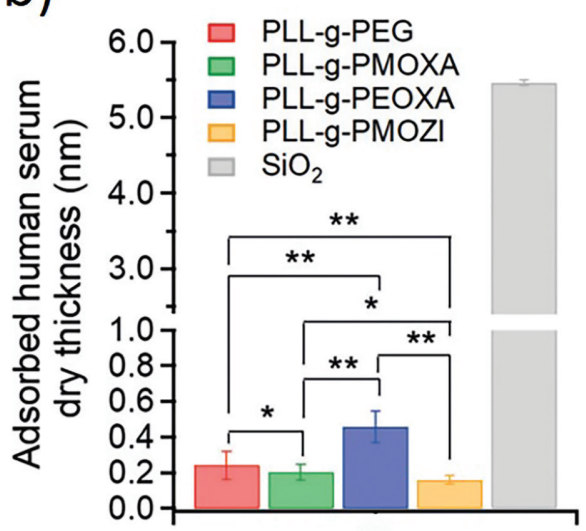

Figure 4 (a) PLL grafted with PEG, PMOXA, PEOXA, and PMOZI antifouling moieties, and (b) the residual adsorbed dry thickness from human serum. Reprinted and adapted with permission from Ref. 153. Copyright 2018 John Wiley and Sons. 
defects than chains (or single molecules) with chemisorbed anchoring units. ${ }^{165}$ Notably, when grafting an increasing number of moieties to a PE (especially when using a weak $\mathrm{PE})$, the number of charges present at the backbone is depleted. Therefore, the adsorption step on an oppositely charged surface is influenced by both the type of grafted unit and the number of charges remaining on the PE chain, determining the coating uniformity and stability. ${ }^{166}$

Both antifouling and receptors/reactive linkers can sometimes have higher affinities for the surface than the PE itself. This can alter the layer conformation, especially at high grafted percentages, possibly resulting in exposure of the backbone and lower surface probe densities than expected. However, for small appended moieties, this kinetic meta-state will be reversed by the entropic and energetic contributions of the electrostatic nature between the charged PE backbone and the surface. ${ }^{36}$ Theoretical work presented by Feuz et al. has described the adsorption mechanism and the stratified display of PLL-PEG on a charged surface ${ }^{166}$ in agreement with previous experimental results. ${ }^{148,167,168}$ By using a Scheutjens-Fleer self-consistent field (SF-SCF) model, they found that the adsorption process was dictated by the molecular architecture. In detail, the grafting ratio $(g)$, the extension of the PEG chains $(D)$, and the ionic strength or Debye length $\left(r_{\mathrm{D}}\right)$ determined the electrostatic and steric contributions to form the film. Figure 5a presents the possible scenarios as a function of these parameters. There was no adsorption for densely grafted PLL with long PEG chains (around 110 ethylene glycol units) due to the steric repulsive effect intrinsic to PEG (last row). Steric effects were present also in the case of shorter PEG chains (approximately 44 ethylene glycol units), and an energy barrier occurred in proximity of the surface, forming a planar diffusive layer (Figure 5a, second row). At lower grafting ratios, more lysine monomers were available, and the positive electrostatic effect was dominant again (Figure 5a, first and third rows). However, stable adsorption was shown only for short PEG chains, where a balance between charged monomers and steric effects allowed the electrostatic driving force to adsorb the PLLPEG at the interface. An energetic contribution was also required from the surface charge density. In the case of uncharged substrates, the PLL-PEG could not readily adsorb and thus did not form uniform coatings. The formation of the layered structure is schematically illustrated in Figure $5 \mathrm{~b}$. Going from left to right, the SF-SCF model predicted the rearrangement of the PEG chains parallel to the normal of the a)

\begin{tabular}{|c|c|c|c|c|}
\hline conditions & sketch & $\begin{array}{l}\text { domi- } \\
\text { nating } \\
\text { regime }\end{array}$ & $\begin{array}{l}\text { shape of interaction } \\
\text { potential }\end{array}$ & interpretation \\
\hline$r_{D} \geq D$ & & \multicolumn{2}{|c|}{ Coulomb U(z) } & \\
\hline$g \Uparrow$ & & $\xi \geq 1$ & D & adsorption \\
\hline
\end{tabular}
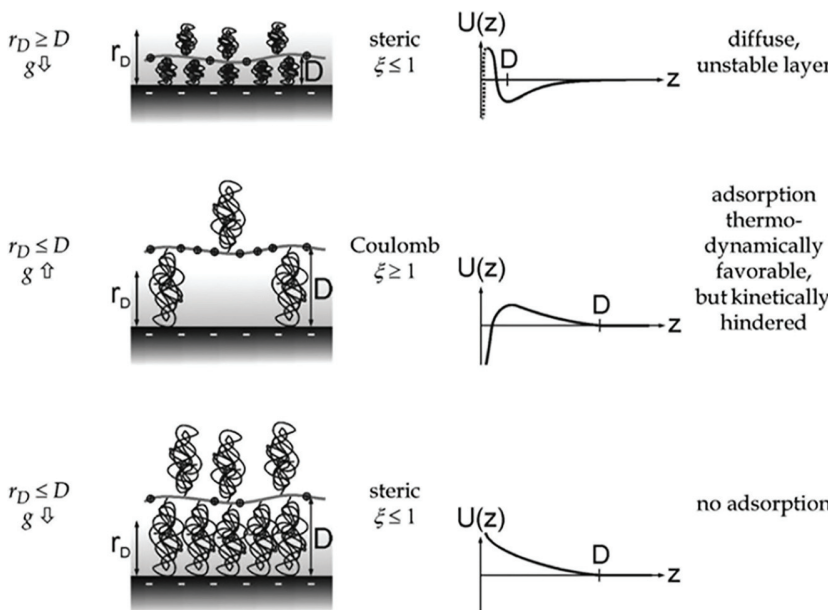

b)
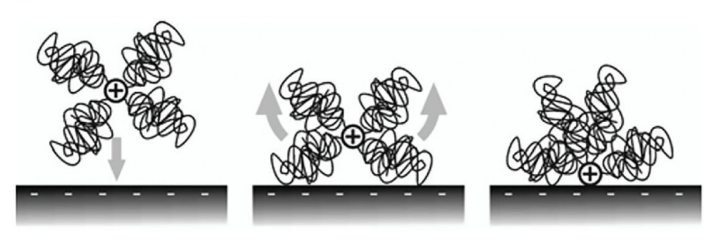

c)
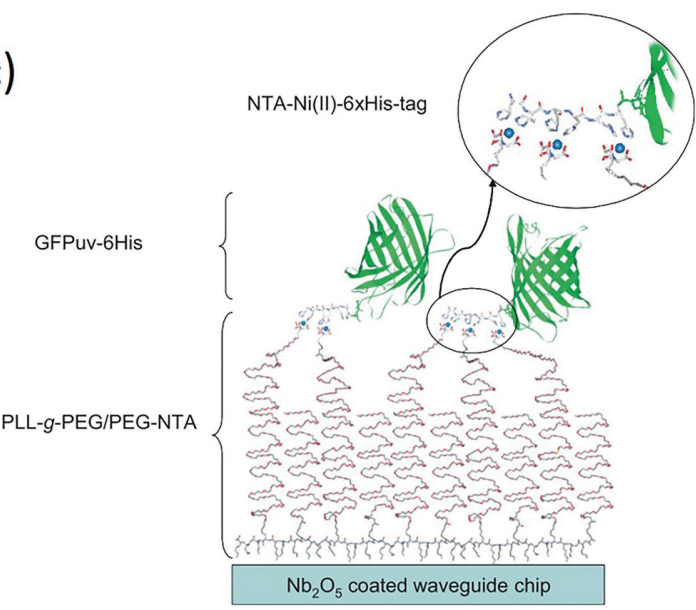

Figure 5 (a) Possible scenarios of the PLL-PEG film formation as a function of $g, D$, and $r_{\mathrm{D}}$, with the dominant driving force, the total potential energy profile, and the following interpretation. (b) Schematic adsorption process of PLL-PEG and the rearrangement of the PEG chains while approaching the surface. Reprinted and adapted with permission from Ref. 166. Copyright 2008 American Chemical Society. (c) Drawing of the envisaged layer structure formed at the interface after adsorption of the modified polyelectrolyte PLL-PEG/PEG-NTA, followed by the loading of $\mathrm{Ni}^{2+}$ ions and the recognition of His-tagged GFP. Reprinted with permission from Ref. 169. Copyright 2005 John Wiley and Sons. 
surface. Once the backbone approached the charged surface, the affinity for the charged lysine units was higher than that for the neutral ethylene glycol moieties. Thus the PEG chains were forced to point outward, forming a layered structure, with the PLL backbone strongly physisorbed at the interface and the PEG chains above it.

Grafting small molecules at the end of the PEG spacer likely does not alter the film formation and its perpendicular directionality. Consequently, the layered architecture will still be formed and maintained under the right conditions ( $g$, $D$, and $r_{\mathrm{D}}$ ). This 3D structure could be conceptually visualized as shown in Figure 5c, where PLL grafted with PEG and PEG end-functionalized with nitrilotriacetic acid (NTA) was adsorbed over a niobium oxide substrate. ${ }^{169}$ The histidine (His) recognition NTA moiety is thought to be on top of the antifouling film, allowing the detection of Histagged macromolecules. In their work, Zhen et al. monitored, by optical waveguide lightmode spectroscopy (OWLS), the full process of PLL-PEG/PEG-NTA adsorption followed by $\mathrm{Ni}^{2+}$ capture and the detection of green fluorescent protein (GFP) tagged with $\mathrm{His}_{6}$. The retention of the NTA biosensing capability at the interface was shown while at the same time preserving the antifouling behavior of the ethylene glycol chains underneath. Such a layered architecture has been applied also with other PE vectors. In a study by Fernandez-Megia and coworkers, a library of chitosan-based polymers for antiadhesive purposes has been described. ${ }^{170}$ Specific bioactive moieties such as biotin, coumarin, cholesterol, and $\alpha$-D-mannose were linked at the end of the PEG chain to promote specific binding while maintaining the antifouling behavior.

In the case of receptors that are more difficult to attach (i.e., charged or with competitive sizes to the grafted units), the orthogonal coupling to a reactive moiety at the end of a spacer displayed on the self-assembled coating seems to be an optimal strategy, reducing the occurrence of preformed molecular complexes or inhomogeneous films. This strategy was used by Huang et al. to form a biorecognition surface based on a mixture of PLL-PEG and PLL-PEG-biotin to detect rabbit immunoglobulin (IgG) and anti-BSA. ${ }^{163}$ After deposition of the biotin-modified PLL, SAv units were anchored until saturation, followed by the binding of the bulky biotinylated receptors, which were either goat anti-rabbit IgG ( $\alpha$ IgG-biotin, $\sim 150 \mathrm{kDa}$ ) or BSA (BSA-biotin, $\sim 66 \mathrm{kDa}$ ), to form the biosensing layer. The same approach was adopted by Städler and coworkers to develop a biorecognition platform to anchor DNA-tagged vesicles at the interface. ${ }^{171}$ Self-assembled polyplexes based on PLL and DNA molecules have been reported to form in solution for use in gene and drug delivery. ${ }^{172}$ Therefore, Städler et al. orthogonally anchored the DNA probe after the functionalized PLL adsorption at the surface. In summary, both approaches based on self-organized architectures with appended receptors or reactive molecules at the end of functional spacers have been widely exploited for biosensing applications and bioengineered coatings depending on the probe size and charge.

Other layered architectures have been described to have biorecognition capabilities on different substrates while at the same time retaining antifouling properties. Duan et al. reduced the length of the ethylene glycol units to four, synthetizing a PLL scaffold grafted with unfunctionalized oligo(ethylene glycol) (OEG) or OEG end-terminated with biotin moieties, forming a silicon-based field effect transistor (FET) for biosensing. ${ }^{90}$ PLL grafted with OEG and OEG end-functionalized maleimide (Mal) groups (PLL-OEG-Mal) was synthesized by us to study multivalent interactions at the interface. After binding a thiolated methyl viologen to the Mal functionality, the heteroternary complex was formed upon consecutive anchoring of cucurbituril and dye-labeled multivalent azopyridines (Figure 6a). ${ }^{161}$ Jeong and coworkers developed an Au surface plasmon resonance (SPR) biosensor for SAv using poly(aspartic acid) (PAsp) bearing a spacer with three ethylene glycol groups and a biotin at the end. ${ }^{173}$ In this case, the PAsp-biotin layer was adsorbed on a bare PLL film, precoated with a thiol SAM. Chen et al. prepared a platform to recognize and anchor cancer cells with expressed folate receptor (FR) on a glass surface. ${ }^{174}$ Folic acid (FA) was grafted at the end to the antifouling PMOXA moiety to form PLL-PMOXA-FA that selfassembled at the surface, displaying FA receptors available for the multivalent binding of the specific HeLa and JEG-3 cell lines (Figure 6b).

\section{Multilayer Architectures with Modified PEs}

Electrostatic interactions provide the main driving force for creating uniform PE coatings. Overcompensation of the charge at the substrate upon adsorption of the PE is responsible for the formation of the so-called PE multilayers (PEMs). ${ }^{175}$ By alternate deposition of PEs bearing opposite charges, multilayer architectures with nano- and micrometer-scale control can be obtained with special characteristics depending on the PEs and their combination. ${ }^{176}$ This layer-by-layer ( $\mathrm{LbL}$ ) assembly procedure was first described in three studies of Decher et al. at the beginning of the 1990s. ${ }^{177-179}$ Thereafter, thousands of studies have been published on the LbL topic, contributing to the development of a facile and customizable method for universal surface functionalization, ${ }^{113,180}$ with applications for optical, biomedical, and bioresponsive materials. ${ }^{77,181}$ The most accepted explanation for the PEM buildup mechanism consists of a continued surface charge inversion by means of overcompensation of charges when adsorbing an oppositely charged PE, which promotes the further adsorption of other PEs. ${ }^{99,182,183}$ Among the variety of parameters to control during the multilayer formation, the salt concentration 


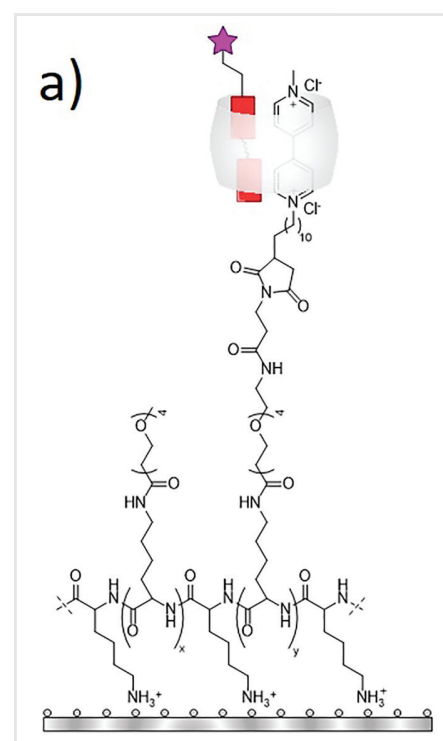

b)

Figure 6 (a) Spatial confinement of the multivalent heteroternary complex of cucurbituril and dye-labeled azopyridine promoted by the anchoring of thiolated methyl viologen on PLL-OEG-Mal passivated $\mathrm{SiO}_{2}$ substrates. ${ }^{161}$ (b) Biorecognition platform to anchor cancer cells expressing FR. The monolayer of PLL-PMOXA-FA was self-assembled at the interface, expressing FA on top of the antifouling layer. After $1 \mathrm{~h}$ of incubation, FR-negative cells could be removed because of a lack of anchoring sites. Reprinted with permission from Ref. 174. Copyright 2015 American Chemical Society.

played a crucial role in determining the roughness and thickness of the target PEM. Both roughness and thickness increase with increasing number of layers and at higher ionic strength, leading to a less homogeneous structure at salt concentrations above 0.5 M. ${ }^{184,185}$ The surface properties of multilayers have been intensively studied with multiple techniques for both wet (e.g., QCM, SPR, and AFM) and dry (e.g., ellipsometry, AFM) states, showing, amongst others, increments in the thickness for each deposited layer. The magnitude of the increases in roughness and thickness strongly depends on the assembly conditions (e.g., ionic strength, $\mathrm{pH}, \mathrm{PE}$ type, and molecular weight). ${ }^{186-190} \mathrm{~A}$ comprehensive description of the LbL properties, applications, and formation mechanism is beyond the scope of this review; the reader is referred to the many existing overviews. ${ }^{113,191-194}$ Here, we focus on the formation of LbL architectures based on at least one PE with appended functional moieties grafted in a preceding synthetic step, with particular interest in their use for making biosensing and engineered interfaces.

Both small and relatively bulky functional groups (e.g., Fc, PEG) appended to the PE have been shown to form various LbL architectures $^{195,196}$ and the structural and mechanical properties depend on the final assembled PEM. Mariani and collaborators formed a bilayer for SAv detection in spiked human saliva samples (Figure 7a). ${ }^{197}$ The biointerface was made of PAH and biotinylated poly(methacrylic acid) (PMAAbiotin) on a porous silicon interferometer (PSi), which showed a 300-fold signal enhancement compared to conventional apparatus such as SPR, a normal interferometer, and an optical fiber. Lower amounts of deposited PE material and thickness were recorded for multilayers of PSS and PAH grafted with guanidinium $(\mathrm{Gu})$ units (PAA-Gu), compared to native PSS/PAA films. ${ }^{198}$ The PSS/PAH-Gu architecture was more rigid and stable at alkaline $\mathrm{pH}$, producing a suitable interface for sensing and water treatment of relevant ions (i.e., $\mathrm{Cl}^{-}$, $\mathrm{SO}_{4}{ }^{2-}$, and $\mathrm{H}_{2} \mathrm{PO}_{4}{ }^{-}$). Wijeratne et al. fabricated functional films based on PAH and poly(2,2-(5-acrylamido-1-carboxypentylazanediyl) diacetic acid) for efficient capture of Histagged proteins on $\mathrm{Au}$ and hydroxylated nylon membranes. $^{199}$ The functionalized multilayers had different thicknesses and swelling capabilities depending on the density of NTA units and the type of metal adsorbed $\left(\mathrm{Cu}^{2+}\right.$ or $\mathrm{Ni}^{2+}$ ), with the application of cost-effective modification of porous membranes for protein purification. The LbL technology can also be used for coating complex 3D ordered structures. Following the pioneering principle of mesoscale self-assembly introduced by Whitesides et al., ${ }^{200}$ the group of Shi functionalized and oriented PDMS-coated scaffolds with targeted modification. ${ }^{201}$ By exploiting poly(diallyldimethylammonium chloride) (PDADMAC) and PAA grafted with either $\beta$-cyclodextrin $(\beta-C D)$ or azobenzene moieties, the PDMS building blocks could be modified at the interface through supramolecular interactions. Introducing another polycation bearing biotin groups (PAH-biotin), they demonstrated the guided formation of ordered 3D architectures with bioactive sites for further loading of biorelevant molecules and potential cell/tissue culture applications.

Recently, PEMs have been reported to express antifouling properties. Regardless of the use of intrinsically 
a) Globally-neutral streptavidin in acetate

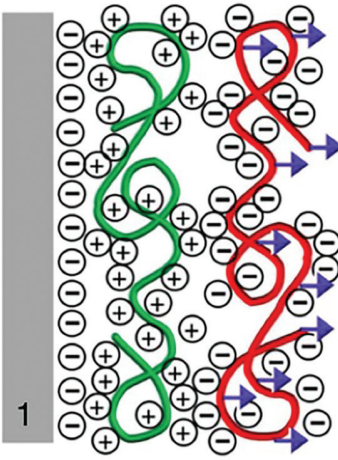

LbL biofunctionalization

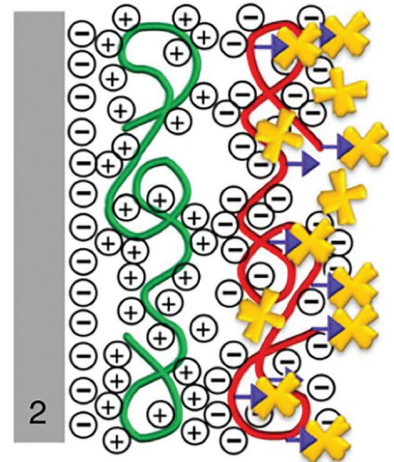

Target binding

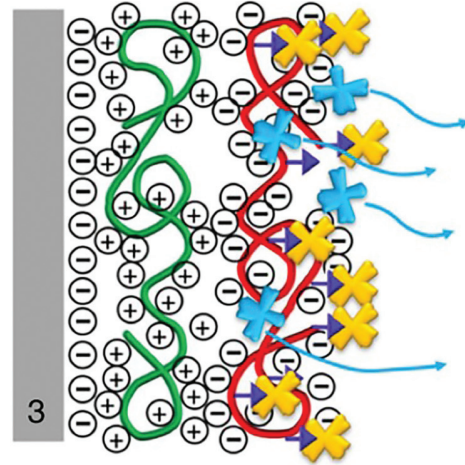

Repulsive unspecific removal

b)

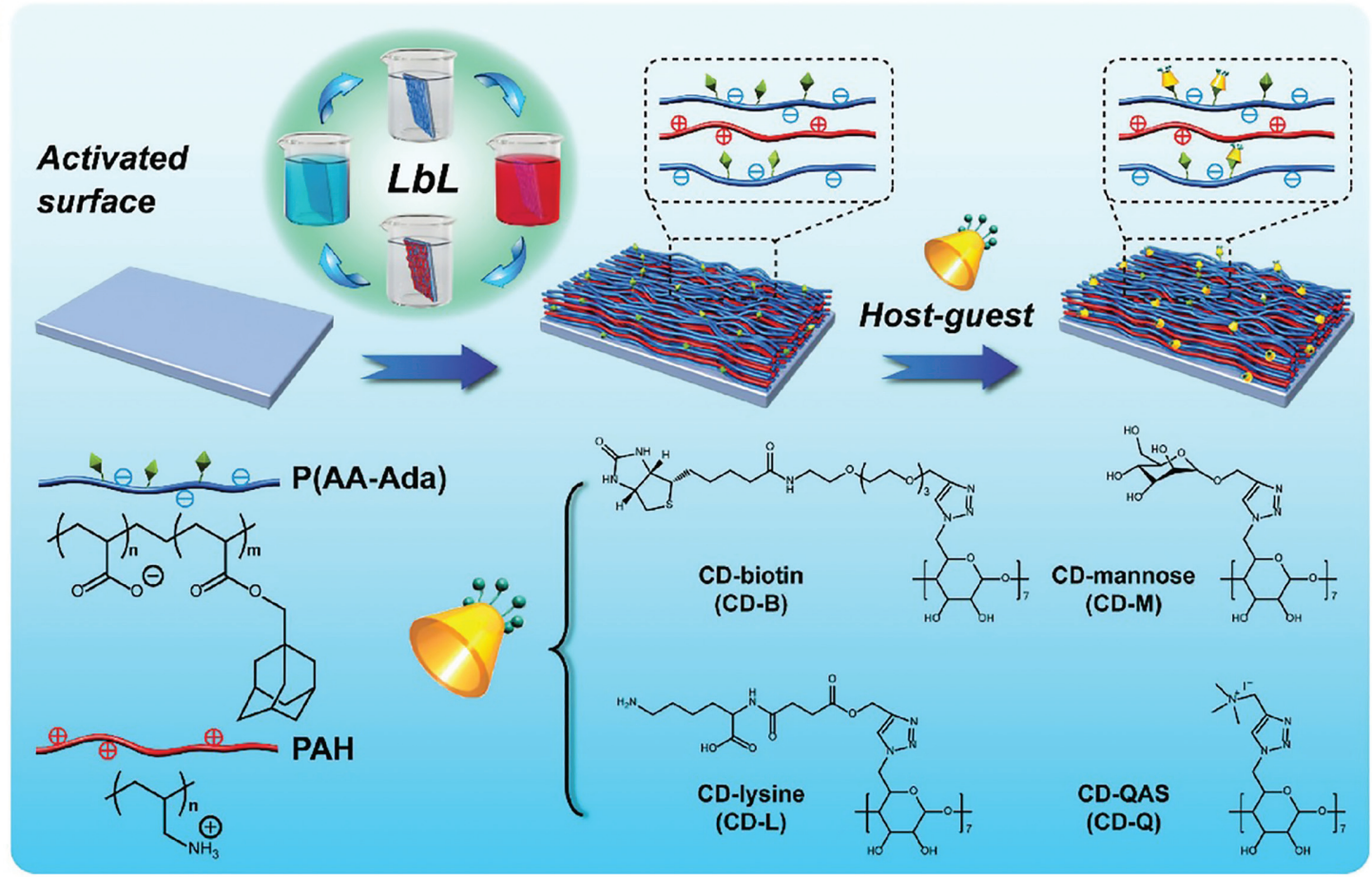

Figure 7 (a) Bilayer formation on a porous silicon interferometer substrate for the nanomolar detection of spiked SAv (yellow) in body fluid (saliva). The green and red lines indicate PAH and PMAA-biotin, respectively. Nonspecifically adsorbed SAv (blue) was desorbed after an acetate/HEPES buffer cycle. Reprinted with permission from Ref. 197. Copyright 2018 Springer Nature. (b) Schematic illustration of the surface functionalization strategy adopted by Cao and coworkers based on the combination of an LbL procedure and supramolecular host-guest interactions. Au, Si, silicon oxide (glass), and polymers (PDMS and polyurethane) were used as substrates for the multilayer formation and the specific subsequent supramolecular modification for biological and biosensing purposes. Reprinted with permission from Ref. 210. Copyright 2016 John Wiley and Sons.

antifouling PEs (i.e., chitosan, alginate, or dextran), ${ }^{202,203}$ as well as the incorporation of nonfouling moieties (e.g., ethylene glycol units), ${ }^{204}$ PEMs have shown to mostly repel protein adsorption. This effect arises from a combination of factors, including the type of PEs, the interactions between the chains inside the multilayer, and the properties of the last layer exposed at the interface. ${ }^{205-208}$ The concept of stoichiometric PEMs introduced by Schlenoff et al. suggests that multifilms have stoichiometric amounts of paired charged groups in the correct spatial proximity ${ }^{209}$ to act as bulk zwitterions or "zwittersolids."22 Cao and coworkers designed a versatile approach for surface modification using 
a combination of $\mathrm{LbL}$ and supramolecular host-guest interactions, which retained antifouling properties. ${ }^{210}$ By alternating depositions of PAA modified with adamantane (Ada) groups (PAA-Ada) and PAH, several bilayers were formed on metal and polymeric substrates with the density of the Ada moieties being controlled by changing number of layers in the LbL process. Fluorescently labeled $\beta-C D$ and BSA were exploited to check the availability of Ada groups, confirming the "open" structure within the multilayer, and to find the optimal antifouling response. Consequently, $\beta$ CDs grafted with functional units such as biotin, mannose, lysine, or quaternary ammonium salt were used, showing potential applications for biosensing and biological purposes (Figure 7b). Antifouling/bioengineered coatings were developed also by Picart et al., investigating the osteoblast adhesion on PEMs composed of PLL and polyanions such as poly-L-glutamic acid (PGA), poly(alginic acid) (PAlg) and poly(galacturonic acid) (PGal). ${ }^{162}$ The antifouling behavior of native architectures (PLL/PGA), (PLL/PAlg) 6 , and $(\mathrm{PLL} / \mathrm{PGal})_{6}$ was compared with the same PEMs either cross-linked or with PGA-RGD as the last layer. Interestingly, the native unmodified PEM structures showed almost zero adhesion even after 8 days, while the rigid (cross-linked) architecture and the ones having RGD moieties provided optimal platforms for cell growth.

\section{Surface Patterning of Modified PEs}

Nano- and micropatterned surfaces have attracted increasing interest for biomedical and biosensing applications. The spatial control of such surfaces is critical for studying cell growth and differentiation, ${ }^{211}$ basic neuronal functions (neuronal networks), ${ }^{212}$ and for forming arrays for protein ${ }^{213}$ or DNA ${ }^{214}$ anchoring/detection. The combination of modified PEs with appended functional moieties and patterning techniques has represented a valuable tool to form cost-effective interfaces with functional micro/nano features.

Among others, modified PLL has been the subject of several studies due to its abilities to be easily functionalized in a preceding synthetic step and to form densely packed homogeneous layers. ${ }^{215}$ Csúcs et al. have used PLL-PEG endfunctionalized with fluorescein (Fl) or RGD (PLL-PEG-Fl and PLL-PEG-RGD) to provide fluorescent microarrays for visualization and detection of cell cultures. ${ }^{211}$ By using microcontact printing $(\mu \mathrm{CP})$, the adhesion and motility of cells were investigated. Due to the combined effect of functional structures backfilled with antifouling PLL-PEG, the motility of epidermal keratocyte was controlled. The distance between the patterned lines affected the lamellipodium protrusion, resulting in the directionality of the movement being parallel to the patterned features. $\mu \mathrm{CP}$ stamps with varying structured features were used to pattern laminin-grafted PLL (PLL-laminin) for locally controlling the axonal outgrowth of hippocampal neurons. $^{212}$ In another example, inverted $\mu \mathrm{CP}$ printing was exploited in a two-step replication process to form 3D microstructures covered with PLL-PEG-biotin or just Fg to investigate the relationship between the shape and functions of single cells. ${ }^{216}$

The group of Textor developed photolithography-based selective molecular assembly patterning (SMAP), ${ }^{217}$ molecular assembly patterning by lift-off (MAPL), ${ }^{218}$ and the locally addressable electrochemical patterning technique (LAEPT), ${ }^{219}$ to selectively confine modified PLL and other functional molecules on various substrates. Patterned architectures were formed at the micro and nanoscale on the surface to study the anchoring of cells, particles, or biomolecules and their response in $2 \mathrm{D}$ and $3 \mathrm{D}$ confined spaces. Figure 8a shows an example of SMAP-formed $\mathrm{TiO}_{2}$ squares within a $\mathrm{SiO}_{2}$ matrix to anchor human foreskin fibroblast cells (HFFs) on $60 \times 60 \mu^{2}$ (top) or $5 \times 5 \mu \mathrm{m}^{2}$ (bottom) features. The formed arrays were functionalized by self-assembled dodecyl phosphate on $\mathrm{TiO}_{2}$, backfilled with PLL-PEG on $\mathrm{SiO}_{2}$, creating a pattern of protein-adhesive regions, surrounded by a protein-resistant background. Thus, immunostained HFF could be locally deposited to better investigate functional mechanisms such as spreading and adhesion (Figure 8a, inset) as a function of the geometry of the array. ${ }^{217}$ The MAPL approach was used to guide the self-organization of larger entities like NPs by using PLLPEG-biotin on both $\mathrm{Nb}_{2} \mathrm{O}_{5}$-patterned substrates and silica NPs using SAv as a linker, with potential application in microarray biosensors (Figure $8 \mathrm{~b}$ ). ${ }^{220}$

Besides PLL, other types of modified PEs and patterning techniques have been used for biosensing applications and engineered coatings. PAH with appended perfluorophenylazide groups was used by Kubo and coworkers to photocouple, among others, 2-O- $\alpha$-D-mannopyranosyl-Dmannopyranose (Man2) at the interface. The Man2 arrays, backfilled with PEOXA, were used to recognize fluorescently labeled lectins such as concanavalin A (Con A). ${ }^{221}$ Patterned structures were formed in combination with LbL architectures. For example, Chien et al. have in an alternate manner deposited PAA coupled with 4-azidoaniline (Az) units (PAA$A z$ ), polyacrylamide, and PAH-PEG (with cyanuric chloride as a linker) to form photo-crosslinked cellular patterns. ${ }^{222}$

\section{Modified PEs for Functional Engineered Coatings and Biosensing}

The deposition of modified PEs gains in importance as a customizable and universal surface modification strategy for different types of substrate materials and micro/nanostructures. Multiple functionalities can be grafted before the surface adsorption, ensuring control 
a)

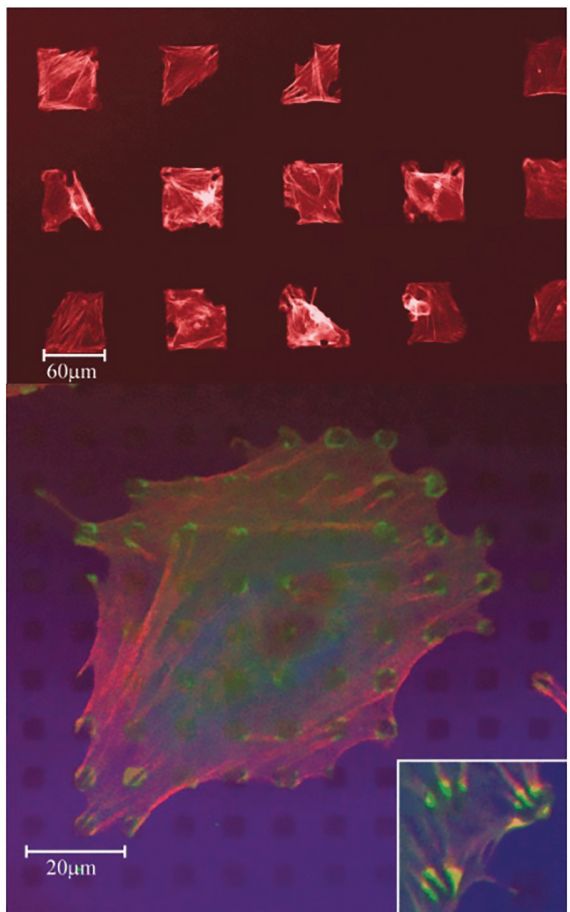

b)
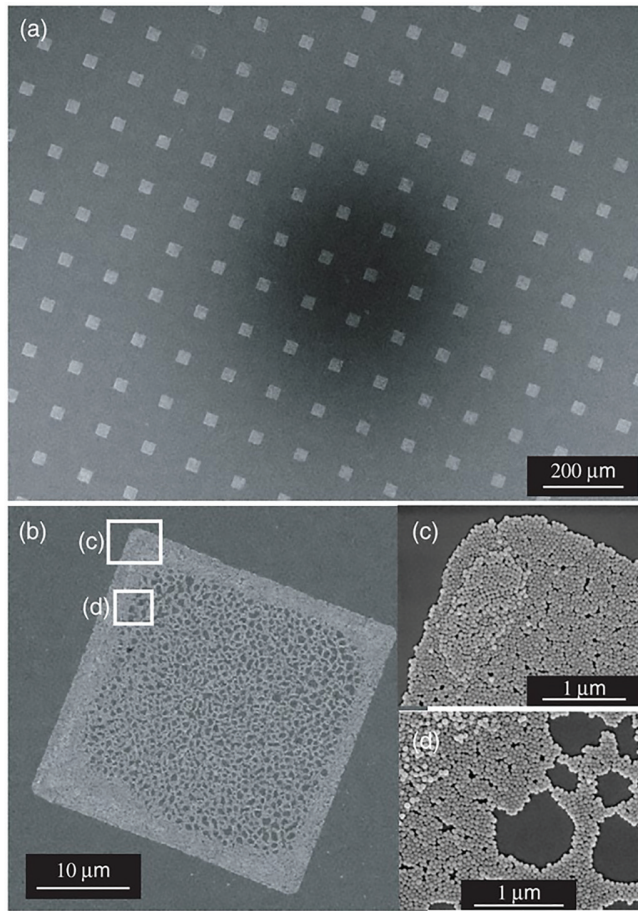

Figure 8 (a) Fluorescence images of immunostained HFF cells on (sub)cellular featured arrays formed by the SMAP technique to study the cell behavior in confined spaces. Reprinted with permission from Ref. 217. Copyright 2002 American Chemical Society. b) Scanning electron microscopy image and magnifications of self-assembled PLL-PEG-biotin-coated silica NPs within a PLL-PEG-biotin microarray formed by MAPL, linked together by a SAv layer. Reprinted with permission from Ref. 220. Copyright 2005 IOP Publishing, Ltd.

over the density and the local surrounding of the appended groups upon assembly at the interface. Tuning both type and density of the receptors/reactive groups at the surface, which respectively affect the selectivity and the sensitivity of the biorecognition event, has opened the possibility of bestowing key features for bioengineered coatings and biosensing applications. Such applications are reviewed in this section, showing the various possibilities offered by the choice of specific components, structures, substrates, and combinations thereof. Particular emphasis is given to the strategies for making antifouling/antibacterial coatings, as well as films for the selective anchoring of cells and other (bio)materials, with an overview of biosensing applications.

\section{Antifouling and Antimicrobial Coatings}

The synthesis of biofunctional modified PEs has been fueled initially by the need for efficient strategies for antifouling coatings. In the preceding section "Monolayers of modified PEs," several chemical moieties anchored to the PE backbone were introduced as an efficient method for reducing nonspecific interactions. Due to the wide adaptability, this strategy has been extensively used for generating antifouling interfaces at bioengineered substrates.
Inspired by nature, Holland and coworkers developed a modified PVAm grafted with dextran units to mimic the external region of a cell membrane, also called the glycocalyx. ${ }^{155}$ By varying the ratio between the dextran and alkanoyl chains, the self-organization on graphite and polyethylene substrates led to highly ordered monolayers with a thickness comparable to that of the PVAm backbone constrained to the surface and with the dextran moieties pointing outward. The highly hydrated dextran suppressed nonspecific interactions after incubation in fresh human plasma. In another study, PVAm-dextran was compared to PVAm-PEG to suppress bacterial adhesion (Staphylococcus epidermidis) on the same substrate. ${ }^{223}$ The PEG-based PE presented less adhesion, probably due to the better shielding of the polyethylene substrate by the PVAm-PEG. The compactness and hydration of PEG chains have been identified as the main properties to fulfill optimal protein repellency. ${ }^{24}$ Owing to the fast and cost-effective coupling of PEG to PEs, PEG-grafted PEs have been synthesized extensively, thus becoming the state-of-the-art for the creation of antifouling interfaces.

Among others, PLL equipped with PEG chains presents the most studied system for both charged and uncharged substrates. As discussed above, PLL-PEG adopts a specific conformation upon surface assembly, with the hydrated ethylene glycol chains perpendicular to the substrate 
(Figure 5b). Such directional and homogeneous layers have excellent antifouling characteristics. The resistance toward the adhesion of cells and proteins has been demonstrated for fibroblast, ${ }^{147,167}$ osteoblast, ${ }^{224}$ Staphylococcus aureus, ${ }^{225}$ serum, ${ }^{226}$ lysozyme and $\alpha$-lactalbumin, ${ }^{148}$ myoglobin, albumin, and Fg. ${ }^{149,227}$ PLL-PEG spontaneously self-organizes on smooth or rough flat surfaces, ${ }^{228}$ on 3D-structured substrates, ${ }^{216}$ on several polymeric materials, ${ }^{229}$ forming surface gradients, ${ }^{230}$ and even on single cells or tissues with $^{231,232}$ or without ${ }^{196}$ added sugar recognition groups, offering multiple ways to control the antifouling properties at the interface. In addition, the high versatility and stability under physiological conditions are the key advantages for exploiting PLL-PEG as a general backfilling strategy. ${ }^{233}$ Microarrays, patterned surfaces, and complex 3D substrates were selectively formed for studying cell spreading, ${ }^{234}$ directionality, ${ }^{235}$ confinement, ${ }^{236,237}$ adhesion behavior, ${ }^{238,239}$ and strength, ${ }^{240}$ as well as for monitoring protein functionalities ${ }^{241}$ and dynamically controlled interactions. ${ }^{213}$ Other antifouling moieties have been grafted to PLL, for example, biomimetic (dextran) ${ }^{156}$ or more efficient (PMOXA) ${ }^{153,242}$ alternatives, contributing to the formation of a plethora of PLL-based strategies for making selforganized nonfouling interfaces.

Despite the popularity of PLL with appended antifouling groups, effort has been increasingly spent on designing approaches that provide antimicrobial functionalities. An original strategy was investigated by Cao and coworkers by the addition of carboxybetaine (CB) to a dextran polymer. ${ }^{243}$ Formation of a biofilm causes a local reduction of $\mathrm{pH}$ in vitro and in vivo. By varying the mole fraction of a synthetic $\mathrm{pH}-$ sensitive $C B,{ }^{244}$ hydrogel interfaces were created with switchable antifouling (cationic form) and antimicrobial (zwitterionic form) characteristics. Brovko et al. described an efficient method for sanitation of food-processing surfaces. ${ }^{245}$ PVAm with tunable appended photoactive dyes, i.e., acriflavine neutral, rose bengal, phloxine $B$, or malachite green, known for their photodynamic bactericidal effect, was deposited on paper as a proof of concept. The antibacterial property was demonstrated for gram-negative and gram-positive bacteria and for yeast microorganisms. Photoinduced cell death rates between 90 and 99\% were observed for paper treated with either PVAm-rose bengal or PVAm-phloxine B upon irradiation, with interesting high selectivity toward bacteria compared to yeast.

Antimicrobial 3D structures were formed by $\mathrm{LbL}$ approaches using engineered PEs. Lee et al. exploited PEI and hyaluronic acid (HA) grafted with catechol units to introduce antibacterial properties to PEMs. ${ }^{180}$ In addition to the improved stabilizing effect, the catechol has latent redox activity that was used to locally nucleate Ag-NPs, thus bestowing bactericidal properties upon Escherichia coli. Varying the type of chemical moieties appended to the last polycation layer allowed Vaterrodt and coworkers to form multifunctional, multilayer structures with antifouling or antibacterial activity. ${ }^{246}$ A film exhibiting a combination of antifouling, antimicrobial, and antibiocorrosion effects was developed by $\mathrm{Xu}$ et al. in a PEM formed by host-guest interactions between PEI- $\beta-C D$ (PEI-CD) and ferrocenemodified chitosan (chitosan-Fc). ${ }^{195}$ The efficiency of antifouling and antimicrobial properties was investigated using gram-positive/negative bacteria, the model microalgae Amphora coffeaeformis (Figure 9), and the arthropod barnacle cyprid, while the antibiocorrosion was investigated by Tafel polarization analysis, showing retention of stability even after 30 days of exposure to seawater.

\section{Platforms for Cell Adhesion, Growth, and Expression}

Exerting multiple degrees of control on cell proliferation, including organization, migration, and adhesion, is of utmost importance for tissue formation/regeneration and organ-on-a-chip development. The use of modified PEs allows the selective display of the type and density of chemical functionalities for engineered biomaterials, locally affecting cell (and culture) evolution.

Modified PEs bearing selected moieties have been applied at the interface to regulate cell expression. Among several integrin-binding domains, the RGD peptide has been exploited as a model sequence for cell-binding studies, due to its high affinity toward integrin receptors ${ }^{247}$ and its resistance to proteolysis. ${ }^{248}$ VandeVondele et al. showed the self-assembly of a modified PE that not only gave protection against nonspecific protein adsorption, but also selective cell adhesion and growth. ${ }^{158}$ The precise tuning of the appended groups (PEG and RGD) was the key aspect for achieving both effects. In conjunction with the high PEG mole fraction, required to maintain the antifouling behavior, the role of the RGD was investigated by monitoring the improved cell adhesion and proliferation on PLL-PEG-RGD compared to PLL-PEG-RDG (nonbinding control sequence). The effect of the RGD surface density was demonstrated by changing the grafted amount from 1 to $58 \%$. Despite the doubled number of fibroblast cells detected for the higher density of RGD, the nonspecific adsorption of serum proteins was 35 times greater at $58 \%$ RGD than at $1 \%$ RGD, confirming the necessity of simultaneously tuning both appended groups. In another study, PLL with varying mole fractions of PEG and RGD was used on Ti surfaces to study the surface physiology and microtopography of osteoblasts. ${ }^{228}$ Comparing the cell behavior on PLL-PEG and PLL-PEG-RGD interfaces, Tosatti et al. demonstrated the nontoxicity of PLL-PEG and the increased integrin binding due to the peptide sequence. Smooth substrates coated with PLL-PEG-RGD presented larger cell numbers than bare ones or substrates coated with PLL-PEG or PLL-PEG-RDG. Rough substrates showed less cell binding than smooth ones, indicating the role of the 

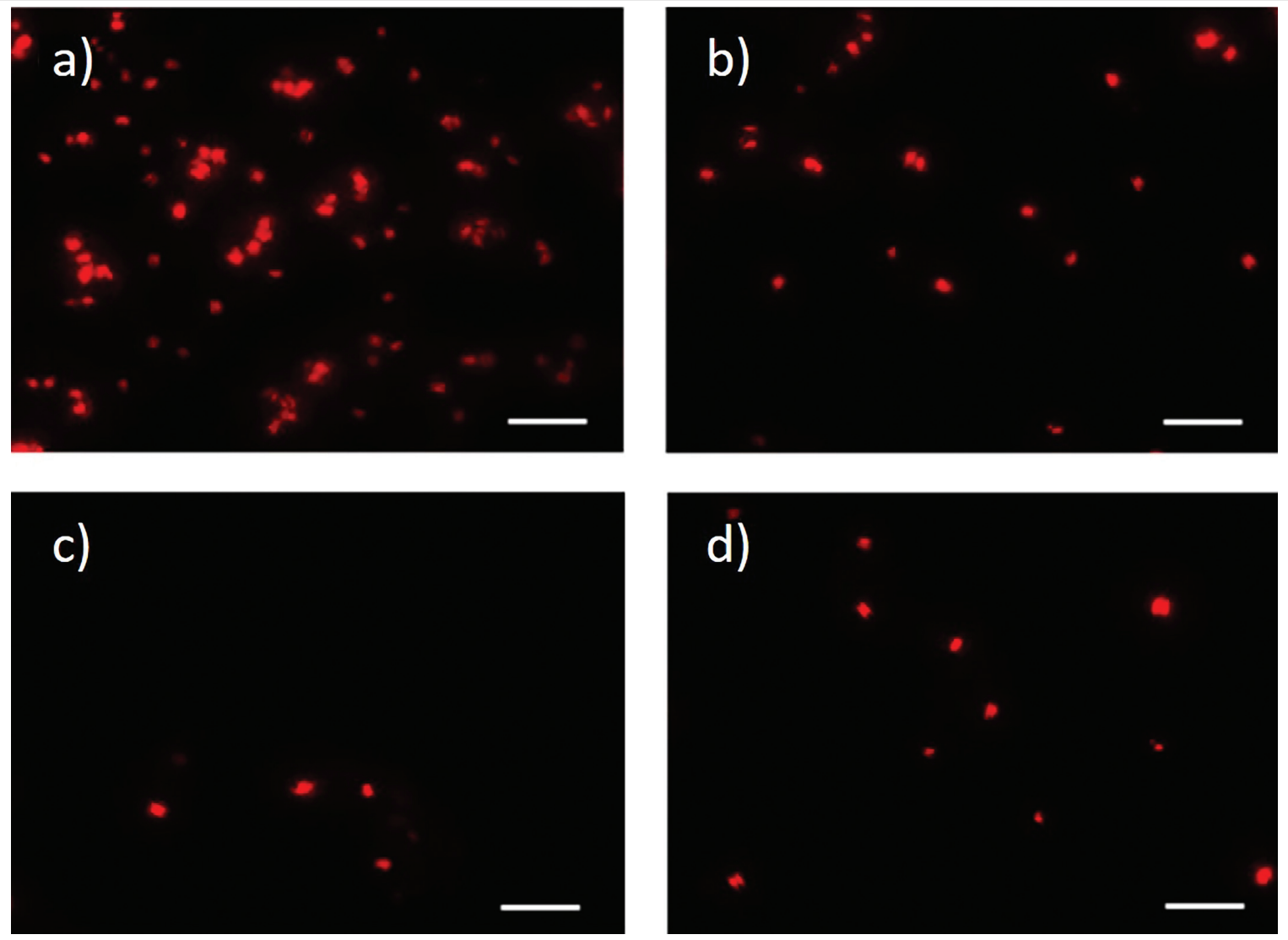

Figure 9 Fluorescence microscopy images of stainless steel: (a) pristine substrate and functionalized ones with (b) 5 and (c) 11 freshly deposited bilayers of PEI-CD/chitosan-Fc and (d) 11 bilayers of PEI-CD/chitosan-Fc aged in seawater for 30 days, showing the resistance to the attachment of Amphora coffeaeformis, after incubation at $25^{\circ} \mathrm{C}$ for $24 \mathrm{~h}$. Scale bars: $50 \mu \mathrm{m}$. Adapted with permission from Ref. 195. Copyright 2016 American Chemical Society.

surface morphology on the cell growth. In some cases, PLLPEG seemed to have a more "osteogenic" effect than PLL-PEGRGD. However, this was probably due to an inhomogeneous PEG layer, creating favorable space for serum protein, which subsequently promoted the cell proliferation. A reduced activity of PEG-RGD chains was recently demonstrated due to small-molecule inclusion such as epigallocatechin gallate (EGCg). ${ }^{249}$ By using a semiempirical quantum-chemical method and experimental data, the EGCg was shown to bind to the ethylene glycol units, blocking the RGD motifs and inhibiting the cell adhesion (Figure 10a). On the other hand, several studies performed in Textor's group $225,228,250-252$ demonstrated the powerful bioselectivity of PLL-PEG-RGD coatings, allowing the anchoring of fibroblasts and osteoblasts, while preventing the adhesion of several bacteria (e.g., S. epidermidis, Pseudomonas aeruginosa, Streptococcus mutans, and S. aureus). In addition to fully covered substrates, PLL-PEG-RGD coatings were also assembled using soft lithography techniques, ${ }^{233}$ with the possibility of forming biointeractive patterns and investigating the local response of single-cell confinement. ${ }^{253}$
RGD and RDG sequences were also grafted to HA, forming a bioalternative to PLL-PEG. Pitt and coworkers described the anchoring of HA grafted with RGD or RDG onto stainless steel by epoxy adhesion and the effect on platelet anchoring and growth. ${ }^{254}$ The nonspecific cell deposition was already visible after $30 \mathrm{~min}$ of incubation in human platelet-rich plasma on the bare surface, but was completely prevented on the HA-coated substrate. On the other hand, HA-RGD restored the spreading of platelets to some extent compared to the nonbinding RDG, showing the modulation of the surface interactions on intrinsically antifouling interfaces.

The group of Marchant synthesized a biomimetic surfactant based on PVAm grafted with hexanal branches, RGD, and maltose (PVAm-RGD-maltose) to mimic the extracellular matrix (ECM) in order to control the diffusion and growth of human microvascular endothelial cells (HMVECs). ${ }^{255}$ In the absence of RGD, no adhesion was observed, due to the antifouling properties of maltose. RGD-rich substrates supported high HMVEC proliferation, displaying a high density of surface-anchoring points compared to RGD-poor ones. However, the migration behavior was reversely affected resulting in 
а)
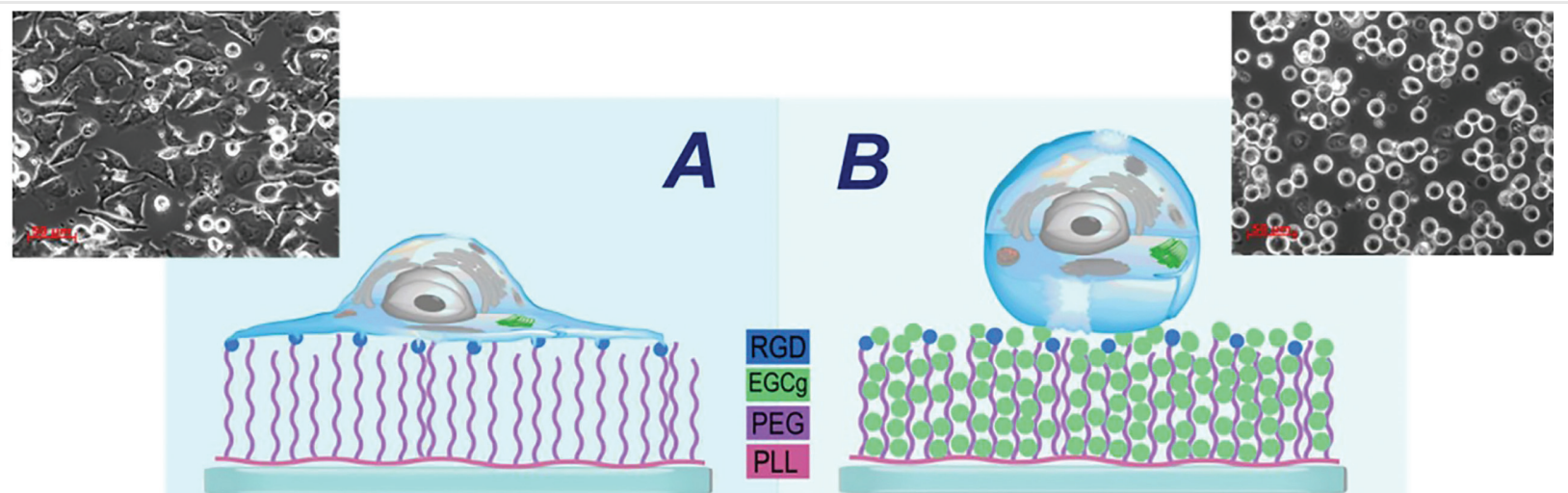

b)
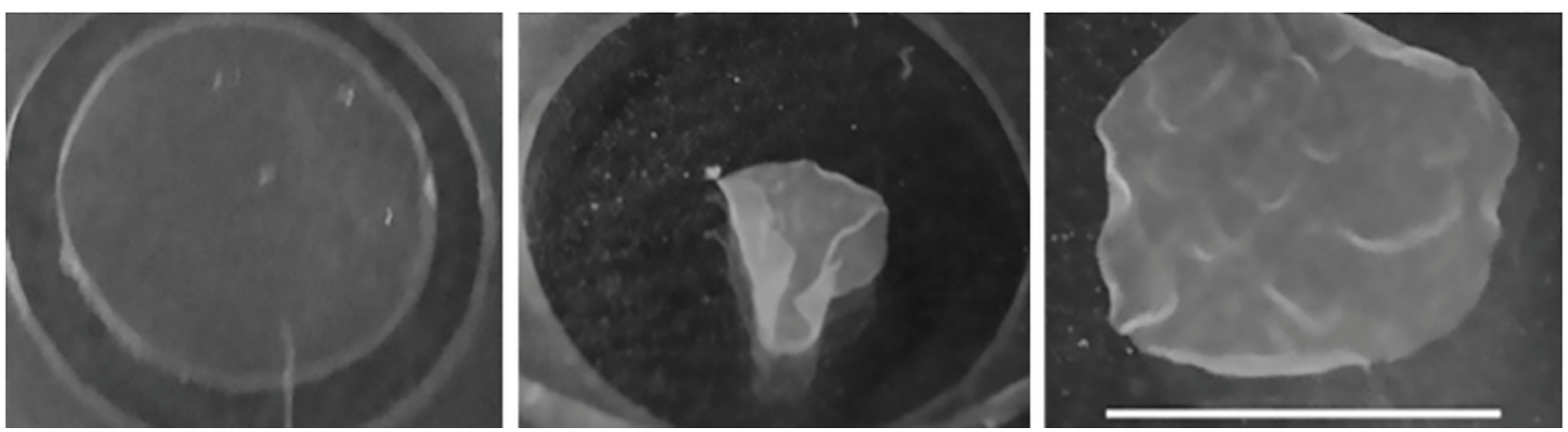

Figure 10 (a) Schematic representation of HeLa cell adhesion and spreading on PLL-PEG-RGD (A) without and (B) with EGCg inclusion, which blocked the RGD motif. The real morphologies were observed by phase-contrast microscopy on the same type of functionalized substrates (insets). Reprinted with permission from Ref. 249. Copyright 2017 Springer Nature. (b) Phase-contrast microscopy images of a 5-day grown fibroblast culture on PLL-PEGRGD-based PEM on an ITO substrate. From left to right: before applying external potential, after application, and recovered cell sheet (scale bar: $1 \mathrm{~cm}$ ). Adapted with permission from Ref. 262. Copyright 2008 John Wiley and Sons.

fixation of HMVEC at their positions, while high mobility was observed for low densities of RGD. By using perfluorinated alkyl chains grafted to the amine groups, PVAm-RGD polymers were self-assembled on expanded polytetrafluoroethylene (ePTFE) substrates as a model to reduce the occlusion and thrombosis after implantation of vascular graft materials. ${ }^{256}$ The biocompatibility of the material was enhanced by stable endothelial attachment, specifically achieved by the PVAmRGD at the interface, with growth rates that were even greater than those seen for native ECM.

Other anchoring sequences, architectures, and functional groups of modified PEs were investigated for cell studies and engineering. For example, IKVAV sequences have been shown to promote spreading, growth, and differentiation of neuronal cells, including adult hippocampal progenitor (AHP) cells. ${ }^{257}$ PLL-PEG grafted with the IKVAV peptide sequence (PLL-PEG-IKVAV), derived from ECM protein laminin, was exploited in combination with IKVAV-modified supported lipid bilayers to study the influence of ligand mobility on cell proliferation. ${ }^{258}$ Despite the larger anchoring area of AHP cells on PLL-PEG-IKVAV, the IKVAV mobility did not influence the cell adhesion and both systems showed a sigmoidal dependence to the IKVAV concentration with an approximate threshold of $8 \mathrm{~nm}$ distance $\left(>3 \mathrm{pmol} / \mathrm{cm}^{2}\right.$ ) for efficient attachment. PAH with appended heparin-binding domains containing the LHRRVKI sequence (PAH-LHRRVKI) was deposited as the last layer on PAH/PAA multilayer structures and compared to similar PEMs having PAH-RGD. ${ }^{259}$ Osteoblast adhesion and functions were monitored by varying the LbL conditions of the PEM build-up. However, the LHRRVKI peptide did not show significant improvement compared to RGD. In a follow-up study, the growth and functionalities of osteoblast MG63 cells on a PAH-RGD-terminated PEM were monitored, showing better proliferation at $\mathrm{pH}$ 6.5, but greater osteogenic abilities (e.g., calcium deposition) at $\mathrm{pH}$ 2 , suggesting an active influence of the PEM architecture. ${ }^{260}$ Cui and coworkers developed a multifunctional, biodegradable PEM to regulate osteogenic activity upon electrical stimulation of preosteoblast MC3T3-E1 cells. ${ }^{261}$ Inspired by the fact that electricity plays a crucial role in living organisms, they grafted tetraaniline, which has a short $\pi$ conjugated system, to PLL or PGA in order to locally stimulate cells by pulsed electrical signals. The 
electroconductive properties of the PEM enhanced the adhesion and proliferation of MC3T3-E1 cells, with improved differentiation into maturing osteoblasts upon electric stimulation, as confirmed by the analysis of ontogenesis-related gene expression. Cell cultures and external electric potential were also applied by Guillaume-Gentil et al. for cell sheet engineering. ${ }^{262}$ The use of PLL-PEG-RGD in an LbL fashion allowed the control of the amount of RGD together with the cell-substrate adhesion forces, forming uniform "tissue-like" coatings and preventing spontaneous detachment. By applying an external positive potential, the defectless "tissue-like" sheet was recovered with the possibility of using it for further applications (Figure 10b).

\section{Modified PEs at Curved Surfaces for Active Targeting and Cell Mimicking}

The controlled synthesis of micro- and nanostructures has caused a scientific revolution for bio- and nanotechnology, paving the way to develop novel strategies for drug delivery, optical devices, photonics, and biosensing applications. Orthogonally displaying biomolecules and responsive functionalities at the interface has been addressed as a crucial aspect for determining the holistic properties of such spherical objects, while at the same time improving their stability in solution. As for flat substrates, the use of modified PEs on particles has emerged as an appealing strategy due to the self-organization at the curved surface and the possibility to customize the colloidal properties. Proper design of the adsorbing PE could ensure control not only of the type and density of moieties expressed at the interface, but also of the outer-layer architecture, affecting the interactions with cells and other biological entities.

As well as the main role of forming camouflaging coatings around microparticles and NPs to reduce the cytotoxicity upon cell/tissue uptake, PEG-grafted PEs have also been used to investigate cell functions, incorporation pathways, and active targeting. Quantum dots (QDs) covered with hyperbranched PEI-PEG were used to study the internalization process in HeLa cells. ${ }^{263}$ The grafting density of PEG on the branched PEI had an influence on the uptake mechanism. At a ratio of four PEG moieties per PEI molecule, compartmentalization inside the organelles was observed, as indicated by the colocalization of light emission from the QDs and the stained organelles. On the other hand, at a ratio of two PEG chains per PEI molecule, fast endocytosis was observed in vesicles followed by slow endosomal escape into the cytoplasm.

The type of modified PE can influence cellular recognition. PS microcapsules with PLL-PEG as the outer layer blocked the phagocytosis of dendritic cells and macrophages more efficiently than PGA-PEG, as reported by
Wattendorf and coworkers. ${ }^{264}$ The PEG chain fraction and length affected the cellular recognition of PS beads. ${ }^{265}$ High PEG (2 kDa) density appended to PLL presented efficient protein resistance but low phagocytosis by dendritic cells and macrophages. Moreover, varying the molecular weight of the PEG chain ( 1 or $5 \mathrm{kDa}$ ) was shown to maintain the protein resistance, but to impair the phagocytosis. The use of such a system for cell targeting was investigated by Faraasen et al. by adding the RGD peptide to the PLL-PEG $\mathrm{PE}^{266}$ By exploiting both PS and poly(D,L-lactic-co-glycolic acid) (PLGA) beads in combination with binding and nonbinding motifs (RGD and RDG, respectively), a specific, receptor-mediated recognition for phagocytosis was identified for PLL-PEG-RGD-covered spheres, with possible applications for specific targeting (Figure 11).

The formation of PLGA carriers for active targeting with specific chemical functionalities was described by Müller and coworkers. ${ }^{267}$ The spontaneous assembly of positively charged PLL-PEG-biotin on negatively charged PLGA spheres produced macroparticles resistant to nonspecific protein adsorption. In addition, the biotin group allowed the localized immobilization of fluorescently labeled SAv at the interface, making it a feasible approach for engineering the coating of biodegradable carriers. Chitosan nanocapsules with appended groups showed a higher active targeting compared to the ones without. $^{268}$ Studies exploiting antibodies directed against TMEFF-2 as a cancer target showed favorable results for chemotherapy ${ }^{269}$ and radiotherapy. ${ }^{270}$ The use of biotin moieties at the end of PEG chains grafted to chitosan allowed the formation of chitosan-PEG-antiTMEFF-2 nanocapsules by means of two-step coupling of avidin and a biotinylated antiTMEFF-2 monoclonal antibody. Despite the promising outcomes and efficient targeting in vitro, the results in vivo were reported to be not efficient.

Empty capsules have been produced using the LbL approach upon dissolution of a sacrificial core, bestowing biotin moieties at the outer layer. The group of Vörös used these systems to study the adhesion energies of PLL-PEGbiotin capsules on SAv-coated surfaces as a model for mimicking biological cells. ${ }^{271}$ In a follow-up study, the morphology of such capsules was studied by varying the multilayer formation procedure, the core material, and the dissolution method, in order to produce stable and proteinresistant scaffolds. ${ }^{272}$ The presence of biotin as the last layer allowed specific recognition of SAv with tunable densities at the interface.

Recently, PS microparticles coated with modified PEs have been proposed to probe and simultaneously analyze the multiplicity of interactions between bacteria and substrates. As a proof of principle, Xie et al. designed two coatings based on PLL grafted with (N-dodecyl-N,Ndimethyl ammonium) butyrate, ${ }^{273}$ which is a bioactive quaternary ammonium compound (QAC) that has shown to 

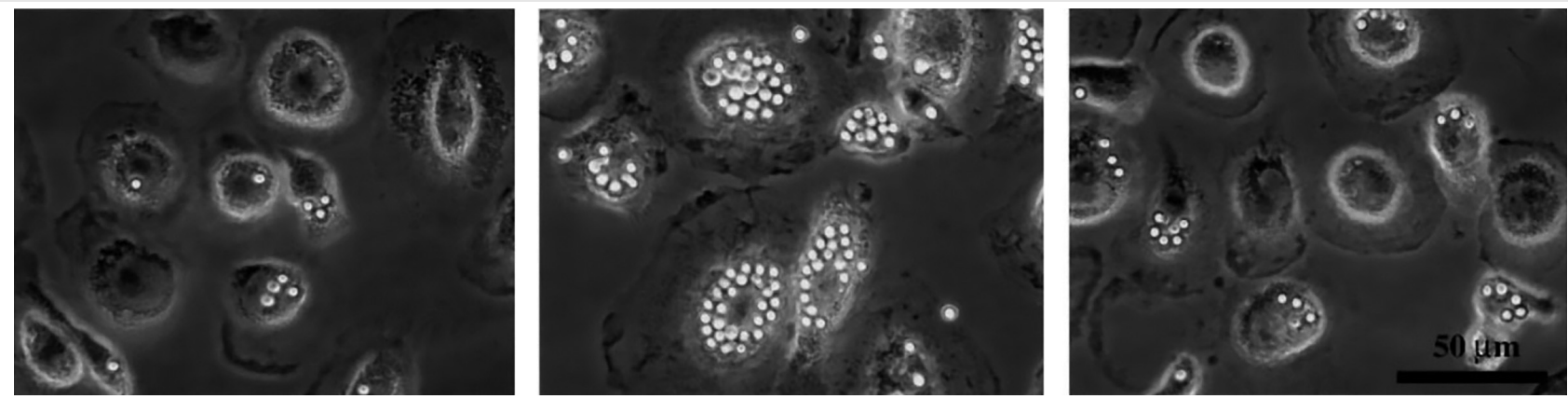

Figure 11 Efficiency of ligand-specific internalization by macrophages for differently functionalized interfaces of PLGA-covered particles. Type of modified PEs used, from left to right: PLL-PEG, PLL-PEG-RGD, and PLL-PEG-RDG (nonbinding). Adapted with permission from Ref. 266. Copyright 2003 Springer Nature.

disrupt the bacterial membrane. ${ }^{274}$ PLL-QAC and PLL-PEG$\mathrm{QAC}$, with the QAC moieties at the end of the PEG chains, were self-assembled on negatively charged PS beads, which were subsequently incubated with $E$. coli. The mixture was then analyzed by flow cytometry, which demonstrated the bactericidal effect of the surface. PLL-PEG-QAC was found to be the most efficient coating in reducing the growth of bacteria at longer time scales. This method showed the potential applications of automatically probing and measuring the effect of different interactions between functionalized substrates and cells, such as bacteria.

\section{Bioengineered Coatings for Arrays of DNA, Proteins, and Vesicles}

Localized positioning of biological materials on surfaces has propelled the advancement of genomics, diagnostics, and biosensing. The multiplexed detection by highthroughput, simultaneous analysis of thousands of parameters requires flexibility of the components in forming the biorecognition interface. Modified PEs have shown compatibility with creating architectures with defined properties, structure, type, and density of receptors or anchoring groups by simple self-assembly. In particular, bio-orthogonal reactive groups have offered further control over the final recognition interface with the possibility of: (i) choosing at will biorecognition elements to be anchored by chemical/biological engineering, (ii) selectively defining recognition areas (often in combination with patterning techniques), (iii) maintaining the properties (e.g., antifouling) and homogeneity of the preorganized architecture, and (iv) rationally improving the selectivity and sensitivity of the biosensing process, regardless of the type of modified PE, substrate material, and detection system. Due to the facile synthesis and availability of the starting materials, the most reported example that employs all of these characteristics is the PLL-PEG-biotin approach.
PLL-PEG-biotin has been exploited by Zhen et al. for anchoring biotinylated engineered $\beta$-lactamase enzymes on niobium oxide substrates. ${ }^{275}$ The architecture formation was monitored stepwise by OWLS, allowing the quantification of the adsorbed mass. The type of the formed layer was investigated by adsorbing either neutravidin (NAv) followed by biotinylated $\beta$-lactamase or preforming a complex NAvlactamase. Only in the latter case, a linear correlation was observed between the amount of surface biotin and the adsorbed mass of both NAv and $\beta$-lactamase (determined by a release process), demonstrating the uniformity of the layered architecture with lactamase on top of the NAv film. The orthogonality of this approach allowed the anchoring of four more variations of engineered $\beta$-lactamase enzymes, while retaining their activities.

Microfluidic systems have been exploited to deliver PLLPEG-biotin PEs for selectively localizing $\mathrm{SAv}^{229}$ or to bind and manipulate bacteriophage $\lambda$-DNA. ${ }^{276}$ After self-assembling the PLL-PEG-biotin layer, anchoring NAv and subsequently attaching a biotinylated DNA probe, $\lambda$-DNA (with an end complementary to the DNA probe) was immobilized in a PDMS microfluidic device for studying interactions with other proteins. In combination with the MAPL technique, a gradient of PLL-PEG-biotin could define patterns with varying densities of active molecules. Morgenthaler et al. showed this concept forming different microarrays of PLLPEG-biotin with decreasing fluorescence intensities after incubation with SAv-Alexa Fluor 488, while at the same time blocking nonspecific protein adsorption. ${ }^{277}$

Due to the feasibility of forming arrays with modified PLLs, numerous efforts have been made on similar analytical protocols that could provide the multiplexity of recognition, performing parallel detection at high speed and accuracy of information. Anchoring of DNA-tagged vesicles was tested on PLL-PEG-biotin surfaces covered with NAv and biotin-DNA. ${ }^{171}$ Microarrays were then formed, studying the colocalized detection of red fluorescent vesicles on biorecognition areas, determined by the patterned green fluorescent SAv. The 
Organic Materials

J. Movilli, J. Huskens

controlled anchoring of vesicles was investigated for promising applications in high-throughput analysis for label-free and selective recognition/amplification processes, ${ }^{278}$ as well as the possibility of delivering micro and nanocompartmentalized environments, for example, as a reservoir for growing nanotubes ${ }^{279}$ or hosting nanoreactors with internalized enzymes. ${ }^{280}$ In further studies, either a spotting technique ${ }^{281}$ or a crossed microfluidic device in concert with MAPL patterning ${ }^{282}$ was used to create selected arrays of anchored vesicles. SAv was bound to a self-assembled PLL-PEG-biotin layer followed by different biotinylated-DNA probes. Functionalized or fluorescently labeled vesicles were selectively immobilized by tagging of the vesicles with complementary DNA (cDNA) sequences (Figure 12). Such approaches were the first steps for the patterning and analysis of membrane proteins, which cannot be usually analyzed in parallel and in cycles involving drying steps. We recently showed the simultaneous formation of different microarrays for multiplexed DNA analysis exploiting PLL-OEG-Mal, while the rest of the substrate exhibited antifouling characteristics owing to the presence of OEG functionalities.

Other types of patterns and modified PEs have been reported for achieving orthogonal anchoring at abiotic and biotic interfaces. Au colloids grafted with thiol DNA complementary to the biotinylated probe were deposited on PLL-PEG-biotin-SAv-coated poly(methyl methacrylate) (PMMA) substrates with remarkable precision. ${ }^{283}$ Nanostripes were formed with different particle surface densities, from single colloid lines to fully connected nanowires, with possible applications in bioelectronics and opticalbased biosensing. ${ }^{284}$ PLL-PEG-NTA arrays were printed for the reversible and selective anchoring of His-tagged proteins, with direct applications in fields such as genomics and proteomics. ${ }^{169}$ PAsp PEs grafted with three ethylene glycol units functionalized with biotin (PAsp-OEG-biotin) were employed to form multilayers with PLL. ${ }^{285}$ Variations of the degree of functionalization and the number of PAspOEG-biotin layers in the PEM were tested, probing the medium distance between anchored SAv proteins and its potential use as an SPR sensing platform. With a similar approach, Harmsma and coworkers developed an optical microring resonator for potential biosensing use by exploiting an LbL approach, having PAH with customizable grafting densities of biotin as the last layer. ${ }^{286}$

A small library of PLLs with short ethylene glycol units was developed, opening new opportunities in biocompati-

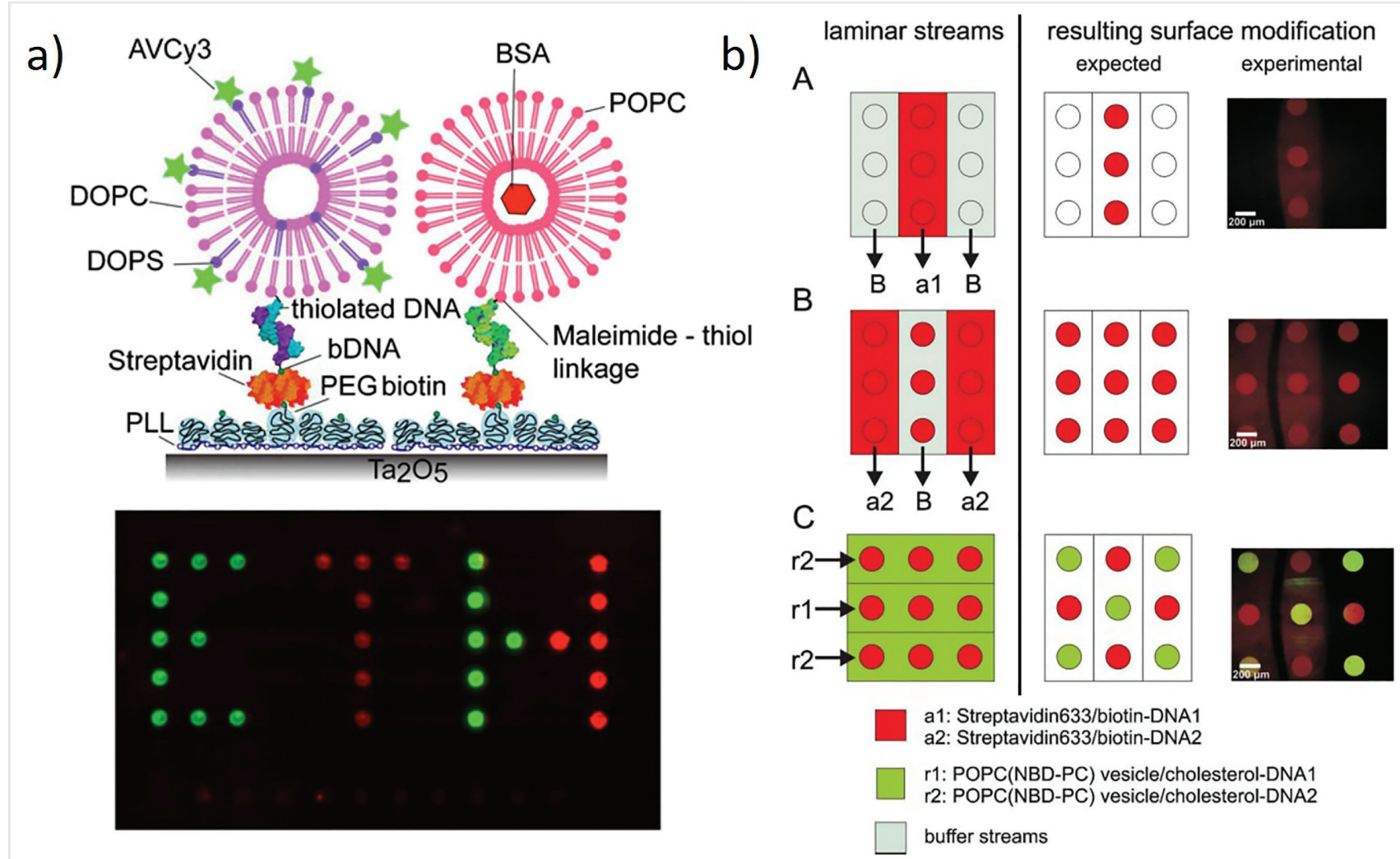

Figure 12 Schematic representations and fluorescence microscopy images of different arrays made with customized vesicles by means of (a) spotting and (b) microfluidic/MAPL patterning techniques. Image (a) has been adapted with permission from Ref. 281. Copyright 2006 AIP Publishing. Image (b) has been adapted with permission from Ref. 282. Copyright 2005. Royal Society of Chemistry. 
ble, combinatorial surface functionalization for biosensing. ${ }^{287}$ Three modified PLLs, namely PLL-OEG-biotin, PLLOEG-azide, and PLL-OEG-hydrazide, were exploited to orthogonally perform bioconjugation with SAv, copperfree click reactions with dibenzyl cyclooctyne moieties, and reversible binding with alginate-aldehyde on isolated pancreatic islet cells. Coadsorption of PLL-OEG-biotin and PLL-OEG-hydrazide displayed a full surface fluorescence overlap when incubated with SAv and alginate-aldehyde with different fluorescent dyes. Another type of click reaction was exploited by us based on the maleimide-thiol reaction. PLL-OEG-Mal deposited on Au substrates was used as an adhesion layer to anchor zwitterionic-like peptides terminated with cysteine, bestowing optimal repellency to both high concentrations of BSA and human plasma samples. ${ }^{164}$

\section{Biosensing Applications}

The type and the density of probes, as well as the layer architecture, determine the target analyte accessibility toward the recognition sites, thus affecting the selectivity and sensitivity of a biosensing surface. The use of modified PEs has shown the feasibility for establishing architectures with specific biorecognition characteristics by molecular design. The main focus of this section is on the biorecognition layer, irrespective of the type of transducer and the detection method (e.g., optical, electrical, and gravimetrical).

Marie and coworkers created selected binding areas on the surfaces of PDMS, PMMA, SU-8, and topas-5013 polymeric materials, by depositing PLL-PEG-biotin by means of microfluidic channels. ${ }^{229}$ Such materials have been abundantly used for fabrication of lab-on-a-chip and microsystem devices based on optical readout. Upon injection of fluorescently labeled SAv, fluorescence emission was detected selectively at the PLL-PEG-biotin areas in less than $10 \mathrm{~min}$, with high sensitivity in the $\mathrm{pM}$ region. Electrochemical avidin sensing was performed by Lepoitevin et al. using a single nanopore functionalized with a PEM having PLL-PEG-biotin as the final layer, ${ }^{288}$ while Marmisollé et al. detected SAv by means of SPR using a monolayer formed by supramolecular interactions based on orthophosphate anions and PAH-biotin. ${ }^{79}$ PEM-coated Ag-NPs with fluorescently labeled PAH and PLL-PEG-biotin as the outer layer have been reported to detect SAv, at concentrations as low as $30 \mathrm{fM}$, by surface-enhanced Raman scattering (SERS). ${ }^{289}$

The use of SAv to link biotinylated receptors has been used for instance by Burg and coworkers in a nanomechanical resonator. ${ }^{290}$ Upon linking anti-goat IgG antibodies engineered with biotin linkers to a PLL-PEG-biotinNAv-passivated resonant cantilever, goat anti-mouse IgGs were detected with a limit of detection (LOD) of $0.7 \mathrm{nM}$ and optimal selectivity compared to the control (no $\operatorname{IgG}$ ) or to human IgG samples (Figure 13a). The same surface chemistry was employed on synchronized QCM and localized SPR (LSPR) chips to detect $\operatorname{IgG}{ }^{291}$ as well as to anchor biotinylated protein A for investigating the immobilization for an enzyme-linked immunosorbent assay (ELISA) in a microfluidic chip. ${ }^{292}$ With this PLL-PEG-biotin-NAv surface functionalization method, a maximum of $40 \%$ enhancement of the immunoactivity ELISA performance was determined compared to other surface immobilization protocols.

Among several factors, the sensitivity of the final binding assay is affected by the combination of the architecture and the detection technique. Duan et al. reported a PLL-OEGbiotin-based FET with an LOD of about $500 \mathrm{fM}$ for SAv ${ }^{90}$ The use of short ethylene glycol chains, consisting of four ethylene glycol units per side chain, was found to be favorable for nano-bioFET applications, as the receptors were kept closer to the transducer surface in comparison to long PEG molecules. In addition, the regeneration of the biorecognition substrate, by flushing with highly acidic buffer, was improved, which is incomplete for PLL-PEG due to its resistance at lower $\mathrm{pH}^{146}$ Davila and coworkers studied the immobilization of biotinylated anti-ovalbumin and the consecutive detection of ovalbumin on various functional PEMs. ${ }^{293}$ By varying both the grafting ratio and the length of the ethylene glycol spacer, a library of PAAOEG-biotin PEs was synthesized and employed as the last adsorbed layer in PEI/(PSS/PAH) multilayers. An optimized platform of PAA-OEG ${ }_{9}$-biotin (25 mol\% with respect to the PE monomer) was used to selectively detect ovalbumin by OWLS and QCM, while retaining optimal antifouling properties. Duan and coworkers exploited PLL grafted with biotin spaced by 4 or 12 ethylene glycol units to sequentially anchor SAv and either biotin-anti-mouse IgG or biotin-anti-human prostate specific antigen (PSA, associated with prostate cancer). Investigating the biosensing capability with QCM, interferometry, and ELISA techniques, nM LODs were reported for PSA in serum and IgG in buffer for PLL-OEG-biotin with the longer OEG spacers. ${ }^{294}$ In a follow-up study, the detection of IgG in serum and PSA in buffer was enhanced by using an acoustic nano-electrochemical system (NEMS) resonator as a biomolecular concentrator at the biorecognition spot (Figure 13b). ${ }^{295} \mathrm{~A}$ 10- and 200-fold decrease of the LOD was found for IgG and PSA, respectively, demonstrating the NEMS resonator applicability with different target molecules and surfacebased biosensing techniques.

The span of detected analytes by means of modified PEs expands beyond proteins and ranges from oligosaccharides to DNA and bigger entities such as cells. Two different types of responsive nanospheres were developed for glucose colorimetric biosensing. Boyer et al. used Au NPs covered with a PEM film and PAA grafted with glucose as the last 
a)
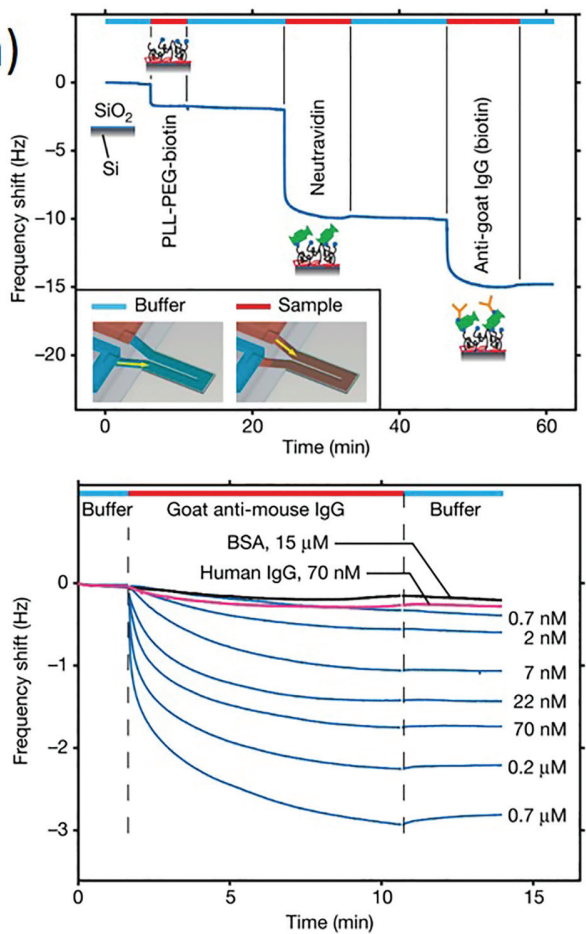

b)
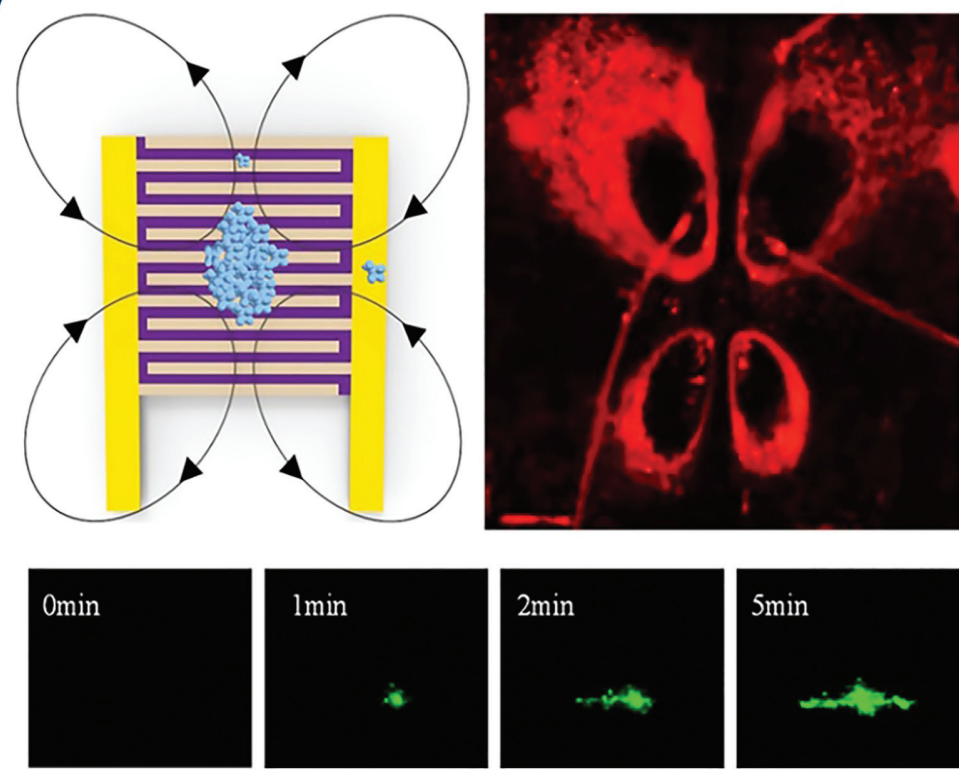

Figure 13 (a) Top: real-time resonance frequency shifts on a $\mathrm{SiO}_{2}$-based cantilever for the consecutive adsorption of PLL-PEG-biotin, NAv, and biotinylated anti-goat $\mathrm{IgG}$, with schematic drawings of the architecture formed at each step. Bottom: QCM frequency shifts for the biorecognition process of goat anti-mouse lgG at different concentrations from $0.7 \mathrm{nM}$ to $0.7 \mu \mathrm{M}$ (blue lines) and controls, in the absence of lgG (black) and in the presence of human IgG. Reprinted with permission from Ref. 290. Copyright 2007 Springer Nature. (b) Top: illustration of NEMS resonator architecture and a fluorescence microscopy image of the microvortices that allowed the concentration of biomolecule at the center of the NEMS device. Bottom: time-lapse fluorescence images of concentrated fluorescently labeled SAv. Reprinted with permission from Ref. 295. Copyright 2018 American Chemical Society.

layer to anchor the lectin Con $A$, which induced the formation of a precipitate. ${ }^{296}$ Upon addition of glucose followed by gentle shaking, the color of the solution changed from transparent to red. In another example, De Geest et al. showed the fast (within $5 \mathrm{~min}$ ) decomposition of multilayer microcapsules formed from a fluorescently labeled polyanion and a phenylboronic acid-based polycation in the presence of glucose. ${ }^{297}$

PAA grafted with ssDNA was exploited by Taira and Yokoyama for DNA chip functionalization. ${ }^{298}$ A second DNA molecule, with the ends complementary to the DNA grafted to PAA and to the target DNA, was hybridized after surface deposition on Au. The addition of this second probe was used to reduce the electrostatic nonspecific interaction in proximity to the surface. The detection of target DNA and single nucleotide polymorphism was assessed by fluorescence spectroscopy, showing good selectivity even at $20 \mathrm{nM}$ concentrations between fully complementary and other sequences. In our group, the systematic variation of the grafting densities of clickable moieties such as maleimide, tetrazine, or dibenzocyclooctyne (Mal, Tz, and DBCO) at the PLL backbone allowed the controlled immobilization of artificial and natural probes for optimized DNA detection (Figure 14a). ${ }^{89,299}$ The PLL backbone was functionalized in a preceding synthetic step, and the ratios of grafted OEG and clickable groups (Mal, Tz, and DBCO; green spheres in Figure 14a) were quantified by NMR. Upon self-assembly of the modified PLL, the surface probe density was controlled exploiting the reaction between the specific moiety appended to the PLL (Mal, Tz, or DBCO) and probes with the complementary click functionality (thiol, transcyclooctene, and azide, respectively; $X$ in Figure 14a). In detail, engineered peptide nucleic acid (PNA) or DNA molecules were anchored to produce DNA biosensing interfaces. The selective DNA hybridization was investigated by means of gravimetric and electrochemical methods, providing an empirical, but at the same time quantitative, prediction of the expected probe density per \% of grafted functional group. Microscale patterns of modified PLL were formed on different polymer substrate materials, exploiting micromolding in capillaries (MIMIC, Figure 14b, left) or a hydrogel-based stamping device (Figure 14b, right). ${ }^{159}$ Multiplexed DNA biorecognition surfaces were created by selectively anchoring thiol-modified probes to the 


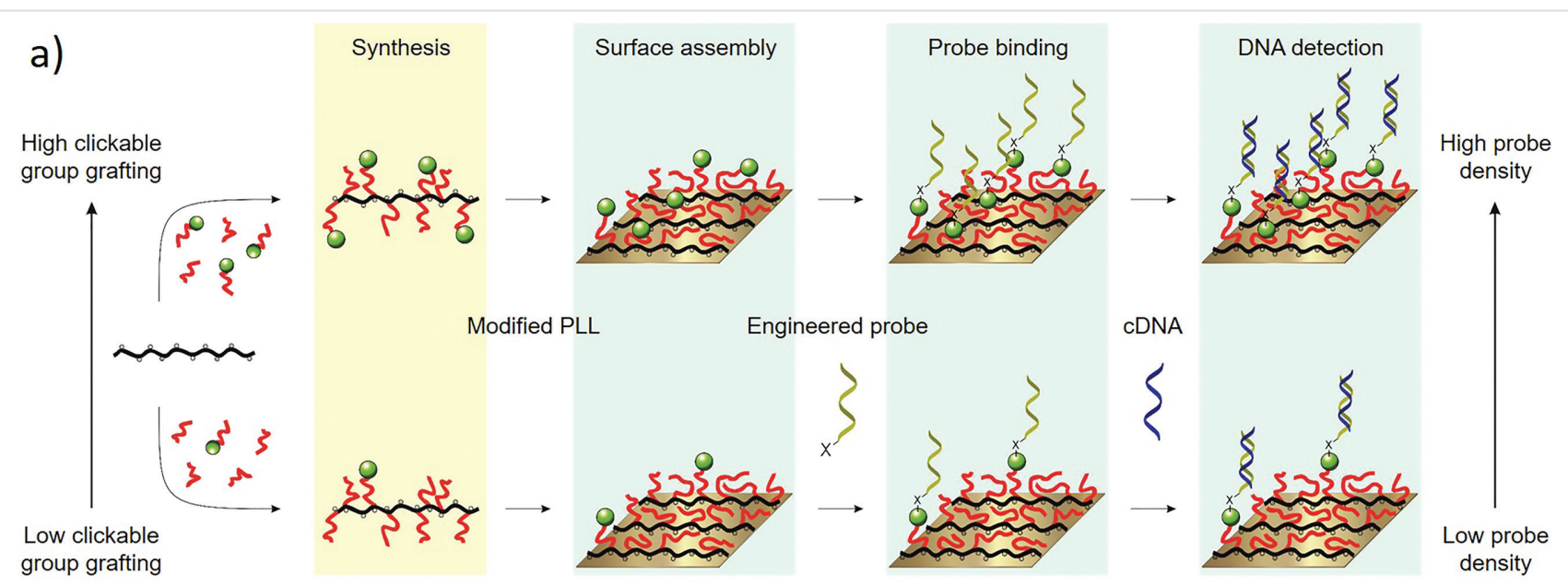

b)
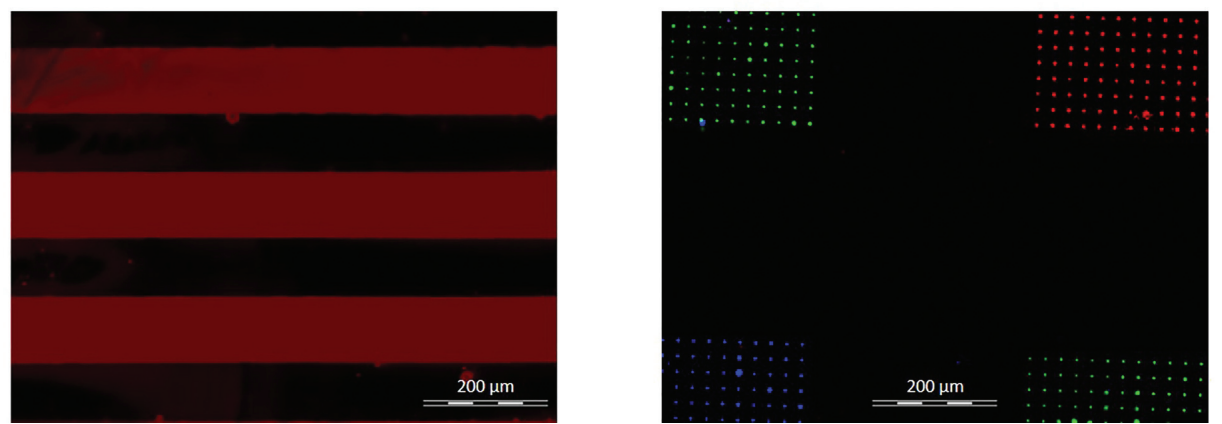

Figure 14 (a) Scheme showing the control of the probe density, during the synthesis of modified PLL with clickable groups (i.e., Mal, Tz, DBCO, green spheres), which provided the target surface density of the engineered probe and the cDNA hybridization at the substrate upon self-assembly of specific modified PLL. Adapted with permission from Ref. 89. Copyright 2018 American Chemical Society. (b) Fluorescence microscopy images of PLL-OEG-Malpatterned polymeric materials (Ormostamp and PDMS, patterned by MIMIC or a hydrogel-based stamping device, respectively) incubated with thiolPNA chains and fluorescently labeled cDNA sequences. Reprinted with permission from Ref. 159. Copyright 2019 American Chemical Society.

maleimide groups at the PLL-functionalized substrates. As a proof of concept, microarrays of three thiol-PNA chains were simultaneously printed, and these probes were capable of selectively detecting fluorescently labeled cDNA sequences from a mixture containing all three cDNA analytes.

Short DNA segments were detected by use of a stratified architecture on noble/ferromagnetic metal particles, with the biotinylated DNA probe anchored to the surface by means of a PLL-PEG-biotin-SAv layer. ${ }^{300}$ Superior quality of the detected signal and the signal-to-noise ratio was observed for magneto-optic SPR detection compared to conventional SPR systems. Gunnarsson et al. lowered the LOD for DNA recognition using short biotinylated DNA probes (15 nt) anchored to a substrate for total internal reflection microscopy by means of PLL-PEG-biotin and a SAv adhesion layer. ${ }^{301} \mathrm{~A}$ sandwich assay was performed allowing the consecutive hybridization of the target DNA molecule (30 nt) and the reporter DNA (complementary to the free end of the target sequence), which was attached to fluorescently labeled vesicles. A recognition signal was detected only in the case of both successful hybridization events, due to the surface proximity of the anchored vesicles, while the vesicles in solution remained "silent." With this approach, an LOD of $10 \mathrm{fM}$ was achieved together with remarkable discrimination of single DNA mismatches by monitoring the vesicle residence time on the surface.

A 3D platform for the capture, detection, and release of specific tumor cells was developed by Hsiao and coworkers. Nanorod arrays were functionalized with PLL-PEG-biotin and SAv for linking the anti-human epithelial cellular adhesion molecule (anti-EpCAM) antibody, specifically used for rare cell isolation. ${ }^{302}$ Efficient immobilization of breast cancer cells MCF7, which express EpCAM, was demonstrated while simultaneously presenting nonadherence to the EpCAM-negative cervical cancer HeLa cell line. Moreover, more than $90 \%$ of captured cells were released by an electrical trigger, without affecting their viability (Figure 15a). Sugai et al. developed a strategy to form one-component arrays for the discrimination of eight types 
a)

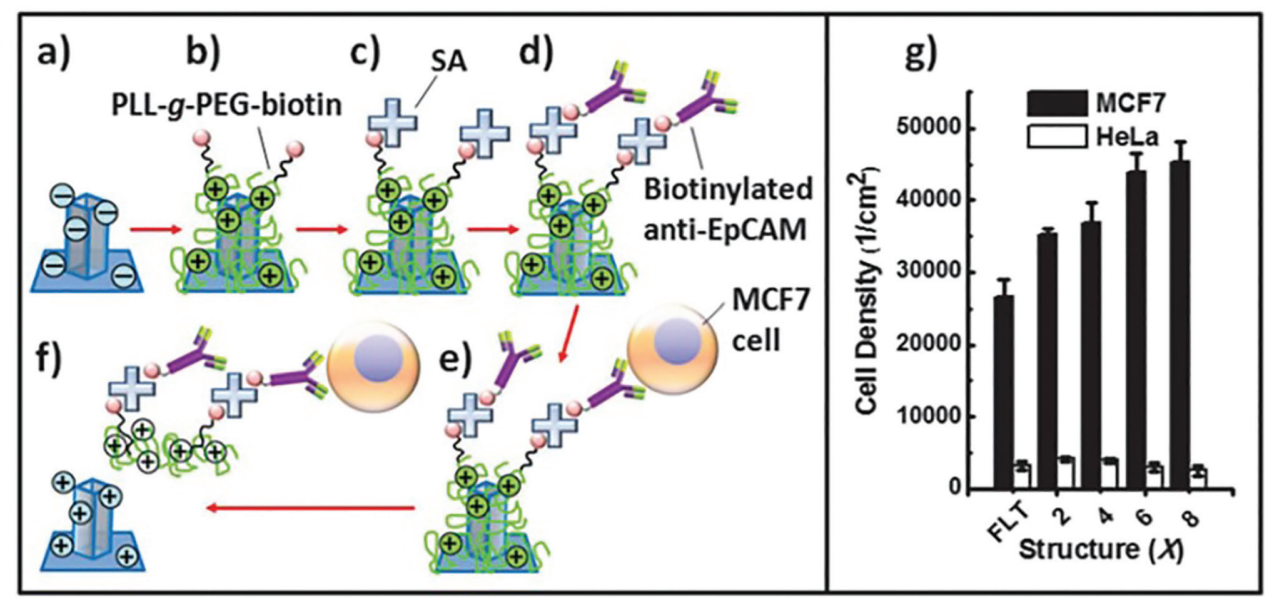

b)

(a)

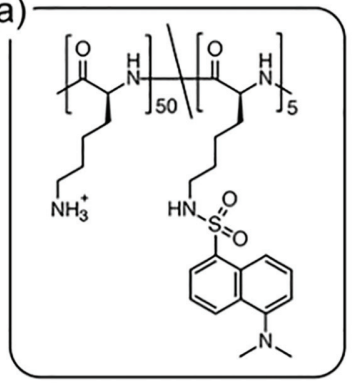

(c)

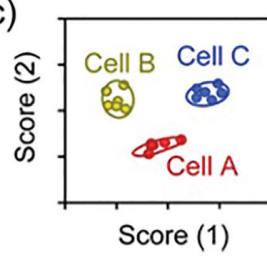

(b)
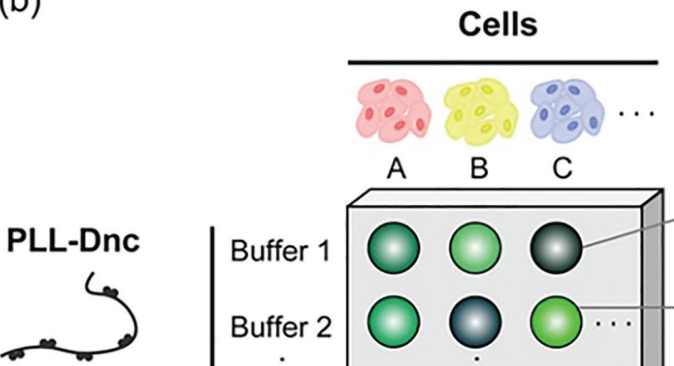

in different buffer solutions

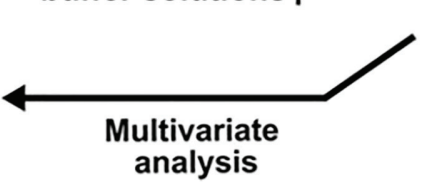

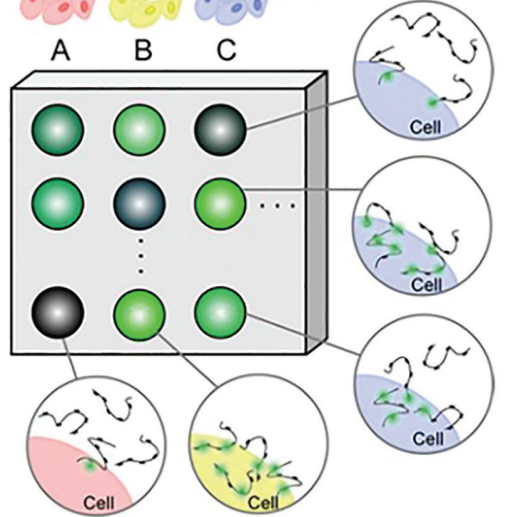

Figure 15 (a) Schematic representation of the stepwise formation of the MCF7 tumor cell-selective nanorod array and the subsequent cell release by an electric stimulus, with a diagram showing the capture yield for EpCAM-positive (MCF7) and EpCAM-negative (HeLa) cell lines. Reprinted with permission from Ref. 302. Copyright 2015 Royal Society of Chemistry. (b) Conceptual figure showing the structure of PLL modified with dansyl functionalities and the one-component assay for discriminating human cells presenting "fingerprint" fluorescence emission due to characteristic interactions between the modified PE and the cell surface, upon changing pH and ionic strength. Reprinted with permission from Ref. 303. Copyright 2019 American Chemical Society.

of human cells (including cells with the same tissue origin, but different subtypes) with high accuracy, by surface adhesion of PLL grafted with dansyl groups, which emit a fluorescence signal in close proximity to hydrophobic regions. ${ }^{303}$ The assay was based on the nonspecific adsorption of the modified PE on the cell surface. Distinct fluorescence signals were detected for different combinations of cell type and adsorbed $\mathrm{PE}$ upon $\mathrm{pH}$ and ionic strength variation, creating a specific fingerprint for each cell line (Figure 15b). Unlike other systems, where the structure of the analyte needs to be known in order to use a correspondent receptor, the use of nonspecific interactions does not require in-depth knowledge of the analyte prior to detection. Exploiting the same PLL with appended dansyl moiety, the group expanded this fingerprinting approach to the detection of other molecules such as proteins, ${ }^{304,305}$ with the possible extension to other biologically relevant entities.

\section{Conclusions}

The engineered display of (bio)molecules at the interface plays a crucial role in defining the characteristics of (a)biotic substrates at the nanoscale level. Among several surface functionalization methods, the strategy of adsorbing 
modified PEs establishes control not only of the type and density of reactive groups but also of the local surroundings, thus defining the physicochemical properties at the interface. Due to the chemical design implemented during a preceding synthetic step, target 2D and 3D architectures can be obtained by self-assembly processes. In combination with patterning techniques, specific functionalities can be introduced at the adsorbed coatings. Strategies based on modified PEs for regulating surface properties have been presented, as well as their applications for bioengineered films and biosensing interfaces. The overview builds on our vision of the use of modified PEs as a functionalization method, focusing on the significant innovations the PEs offer to surface modification methodologies. The use of modified PEs has introduced the advantage of covalently binding chemical functionalities in a preceding synthetic step to tune the displayed biomolecules at the interface, regardless of the substrate material, enhancing the reproducibility of the expressed feature. Such degrees of control are highly valuable for producing biorecognition devices.

The PE adsorption mechanism has been discussed to describe the variety of chemical moieties that can be grafted to PE backbones. PE adsorption processes yield mono, multi, and patterned layer assemblies at interfaces. Augmented characteristics of the formed structures, stemming from the combination of functionalities and the type of architecture used, have been reviewed. Examples of the use of modified PEs for the development of functional engineered coatings and biosensing purposes have been extensively analyzed. The antifouling and antimicrobial characteristics, which are fundamental aspects in materials sciences and surface chemistry, have been discussed. In addition to these features, modified PE-functionalized platforms to promote orthogonal anchoring of probes and biological entities have been investigated. Reactive/affinity groups (e.g., peptides, proteins, and nucleotides) displayed on flat and curved surfaces have been presented to selectively immobilize, pattern, and detect, among others, antibodies, proteins, DNA, vesicles, and cells, showing the applicability for cell studies, active targeting, and biosensing, extending the window of applicability of modified PE-based devices.

The strategy of using modified PEs for addressing specific, holistic functionalities at interfaces has played a pivotal role in developing surface functionalization approaches, affecting multidisciplinary aspects in materials sciences at macro and nanoscale levels. Due to the ease of synthesis, the variety of appended groups, and the wide substrate applicability, modified PEs can be exploited to further expand the range of (bio)applications. The programmed functionalities expressed by the combination of synthetic design and surface architecture give additional degrees of control for producing platforms tailored for studying biological processes and genetic expressions, testing new drugs and probing interactions between selected biomolecules. The adsorption of PLL modified with appended groups is, by far, the most used and characterized methodology. The wide adaptability and the straightforward functionalization procedures have led to a variety of engineered PLL-based interfaces and devices for biological purposes and biorecognition applications. However, the majority of the examples reported were performed in standardized aqueous solutions. The absence of systematic studies exploiting complex biological fluids has prevented the pursuit of further biomedical validation or clinical trials.

The versatile use of modified PEs could potentially enhance the state-of-the-art of biosensing equipment. Devices for studying (biologically) relevant interactions and chemical pathways are still limited to common transducer materials and chemical functionalities of the probes. Customized devices with improved quantification properties can be formed by simply engineering the PE molecule of choice. The challenge that follows for such PEbased systems would be their integration in next-generation (bio)sensor platforms, capable of detecting single entities efficiently and cost-effectively, simultaneously advancing the production of future technologies, such as an artificial nose/tongue and units for storing/reading biobased data. The possibility of choosing the grafted components, interfacial architecture, and the type of detection system could be exploited to improve patientpersonalized, multiplexed, and ultrasensitive biosensor devices. Consequently, genetic diseases, treatments, drug tests, and immune responses could be rapidly detected and monitored. Surface modification by purposefully functionalized PEs can therefore be exploited by (bio)chemists, (bio) engineers, and biologists as a generic tool for the functionalization of highly customizable platforms at the micro- and nanoscale levels to study virtually any type of (bio)interaction.

\section{Abbreviations}

$\begin{array}{ll}\text { Ada } & \text { adamantane } \\ \text { AHP } & \text { hippocampal progenitor } \\ \text { AMA } & \text { aminoethyl methacrylate } \\ \text { Az } & \text { 4-azidoaniline } \\ \text { BSA } & \text { bovine serum albumin } \\ \text { CB } & \text { carboxybetaine } \\ \text { CD } & \text { cyclodextrin } \\ \text { Con A } & \text { concanavalin A } \\ \text { COP } & \text { cyclic olefin polymer } \\ \text { DBCO } & \text { dibenzocyclooctyne } \\ \text { ECM } & \text { extracellular matrix } \\ \text { EGCg } & \text { epigallocatechin gallate } \\ \text { EHEC } & \text { ethyl hydroxyethyl cellulose } \\ \text { ELISA } & \text { enzyme-linked immunosorbent assay }\end{array}$


EpCAM epithelial cellular adhesion molecule

ePTFE

FA

FC

FET

$\mathrm{Fg}$

Fl

FR

GFP

HA

HFF

HMVEC

IgG

ITO

LAEPT

LbL

LOD

LSPR

Mal

Man2

MAPL

NAv

NEMS

NP

NTA

OEG

OWLS

PAA

PAAm

PAH

PAlg

PAOXA

PAsp

PDADMAC

PDMS

PE

PEG

PEI

PEM

PEOXA

PGA

PGal

PLGA

PLL

PMAA

PMMA

PMOXA

PMOZI

PS

PSA

PSi

PSS

PTFE

expanded polytetrafluoroethylene

folic acid

ferrocene

field effect transistor

fibrinogen

fluorescein

folate receptor

green fluorescent protein

hyaluronic acid

human foreskin fibroblast

human microvascular endothelial cells

immunoglobulin

indium tin oxide

locally addressable electrochemical pattern-

ing technique

layer-by-layer

limit of detection

localized SPR

maleimide

2-O- $\alpha$-D-mannopyranosyl-D-mannopyranose molecular assembly patterning by lift-off neutravidin

nano-electrochemical system

nanoparticle

nitrilotriacetic acid

oligo(ethylene glycol)

optical waveguide lightmode spectroscopy

poly(acrylic acid)

poly(allylamine)

poly(allylamine hydrochloride)

poly(alginic acid)

poly(2-alkyl-2-oxazoline)

poly(aspartic acid)

poly(diallyldimethylammonium chloride)

poly(dimethylsiloxane)

polyelectrolyte

poly(ethylene glycol)

poly(ethylene imine)

polyelectrolyte multilayer

poly(2-ethyl-2-oxazoline)

poly-L-glutamic acid

poly(galacturonic acid)

poly(D,L-lactic-co-glycolic acid)

poly-L-lysine

poly(methacrylic acid)

poly(methyl methacrylate)

poly(2-methyl-2-oxazoline)

poly(2-methyl-2-ozazine)

polystyrene

prostate specific antigen

porous silicon interferometer

poly(sodium 4-styrenesulfonate)

poly(tetrafluoroethylene)

\begin{tabular}{|c|c|}
\hline PVAm & poly(vinyl amine) \\
\hline PVP & poly(2-vinylpyridine) \\
\hline QAC & quaternary ammonium compound \\
\hline QCM-D & $\begin{array}{l}\text { quartz crystal microbalance with dissipation } \\
\text { monitoring }\end{array}$ \\
\hline QD & quantum dot \\
\hline SAM & self-assembled monolayer \\
\hline SAv & streptavidin \\
\hline SERS & surface-enhanced Raman scattering \\
\hline SF-SCF & Scheutjens-Fleer self-consistent field \\
\hline SMAP & selective molecular assembly patterning \\
\hline SPR & surface plasmon resonance \\
\hline ssDNA & single-stranded DNA \\
\hline $\mathrm{Tz}$ & tetrazine \\
\hline$\mu \mathrm{CP}$ & microcontact printing \\
\hline
\end{tabular}

\section{Funding Information}

The Horizon 2020 Health project "ULTRAPLACAD" (nr. 633937) is acknowledged for financial support.

\section{References}

(1) Koberstein, J. T. MRS Bull. 1996, 21, 16.

(2) Stamm, M. Polymer surfaces and interfaces characterization technique. In: Polymer Surfaces and Interfaces Characterization, Modification and Applications. Stamm, M., ed.; Springer: Berlin, 2008, 1-16.

(3) Costello, M. T. Corrosion inhibitors and rust preventatives. In: Lubricant Additives: Chemistry and Applications, 2nd edn. Rudnick, L. R., ed.; CRC Press: Boca Raton, 2009, 421-443.

(4) Torlak, E.; Sert, D. Int. J. Biol. Macromol. 2013, 60, 52.

(5) Krebs, F. C. Sol. Energy Mater. Sol. Cells 2009, 93, 394.

(6) Gosselin, E. A.; Eppler, H. B.; Bromberg, J. S.; Jewell, C. M. Nat. Mater. 2018, 17, 484

(7) Leslie, D. C.; Waterhouse, A.; Berthet, J. B.; Valentin, T. M.; Watters, A. L.; Jain, A.; Kim, P.; Hatton, B. D.; Nedder, A.; Donovan, K.; Super, E. H.; Howell, C.; Johnson, C. P.; Vu, T. L.; Bolgen, D. E.; Rifai, S.; Hansen, A. R.; Aizenberg, M.; Super, M.; Aizenberg, J.; Ingber, D. E. Nat. Biotechnol. 2014, 32, 1134.

(8) Park, J.; McShane, M. J. ACS Appl. Mater. Interfaces 2010, 2, 991.

(9) Abraham, S.; Brahim, S.; Ishihara, K.; Guiseppi-Elie, A. Biomaterials 2005, 26, 4767.

(10) Love, J. C.; Estroff, L. A.; Kriebel, J. K.; Nuzzo, R. G.; Whitesides, G. M. Chem. Rev. 2005, 105, 1103.

(11) Gagner, J. E.; Shrivastava, S.; Qian, X.; Dordick, J. S.; Siegel, R. W. J. Phys. Chem. Lett. 2012, 3, 3149.

(12) Bertrand, N.; Leroux, J. C. J. Control. Release 2012, 161, 152.

(13) Mura, S.; Nicolas, J.; Couvreur, P. Nat. Mater. 2013, 12, 991.

(14) Cao, Z.; Yu, Q.; Xue, H.; Cheng, G.; Jiang, S. Angew. Chem. Int. Ed. 2010, 49, 3771.

(15) Chen, Y.; Han, H.; Tong, H.; Chen, T.; Wang, H.; Ji, J.; Jin, Q. ACS Appl. Mater. Interfaces 2016, 8, 21185.

(16) Veronese, F. M.; Pasut, G. Drug Discov. Today 2005, 10, 1451. 
(17) Knop, K.; Hoogenboom, R.; Fischer, D.; Schubert, U. S. Angew. Chem. Int. Ed. 2010, 49, 6288.

(18) Viegas, T. X.; Bentley, M. D.; Harris, J. M.; Fang, Z.; Yoon, K.; Dizman, B.; Weimer, R.; Mero, A.; Pasut, G.; Veronese, F. M. Bioconjugate Chem. 2011, 22, 976.

(19) Kanazaki, K.; Sano, K.; Makino, A.; Homma, T.; Ono, M.; Saji, H. Sci. Rep. 2016, 6, 33798.

(20) Selvestrel, F.; Moret, F.; Segat, D.; Woodhams, J. H.; Fracasso, G.; Echevarria, I. M. R.; Baù, L.; Rastrelli, F.; Compagnin, C.; Reddi, E.; Fedeli, C.; Papini, E.; Tavano, R.; MacKenzie, A.; Bovis, M.; Yaghini, E.; MacRobert, A. J.; Zanini, S.; Boscaini, A.; Colombatti, M.; Mancin, F. Nanoscale 2013, 5, 6106.

(21) Byrne, J. D.; Betancourt, T.; Brannon-Peppas, L. Adv. Drug Deliv. Rev. 2008, 60, 1615.

(22) Schlenoff, J. B. Langmuir 2014, 30, 9625.

(23) Harris, J. M. Introduction to biotechnical and biomedical applications of poly(ethylene glycol). In: Poly(Ethylene Glycol) Chemistry Biotechnical and Biomedical Applications. Harris, J. M., ed.; Springer: Boston, 1992, 1-14.

(24) Wang, R. L. C.; Kreuzer, H. J.; Grunze, M. J. Phys. Chem. B 1997, 101, 9767.

(25) Konradi, R.; Acikgoz, C.; Textor, M. Macromol. Rapid Commun. 2012, 33, 1663.

(26) North, S. H.; Lock, E. H.; Taitt, C. R.; Walton, S. G. Anal. Bioanal. Chem. 2010, 397, 925.

(27) Rogers, K. R. Mol. Biotechnol. 2000, 14, 109.

(28) Knoll, W.; Park, H.; Sinner, E. K.; Yao, D.; Yu, F. Surf. Sci. 2004, $570,30$.

(29) Moon, J. H.; Shin, J. W.; Kim, S. Y.; Park, J. W. Langmuir 2002, 12, 4621.

(30) Saha, B.; Evers, T. H.; Prins, M. W. J. Anal. Chem. 2014, 86, 8158.

(31) Saerens, D.; Huang, L.; Bonroy, K.; Muyldermans, S. Sensors 2008, 8, 4669.

(32) Biagiotti, V.; Porchetta, A.; Desiderati, S.; Plaxco, K. W.; Palleschi, G.; Ricci, F. Anal. Bioanal. Chem. 2012, 402, 413.

(33) Peterson, A. W.; Heaton, R. J.; Georgiadis, R. M. Nucleic Acids Res. 2001, 29, 5163.

(34) Brecht, A.; Gauglitz, G. Biosens. Bioelectron. 1995, 10, 923.

(35) Zhang, S.; Wright, G.; Yang, Y. Biosens. Bioelectron. 2000, 15, 273.

(36) Reimhult, E.; Höök, F. Sensors 2015, 15, 1635.

(37) Petryayeva, E.; Krull, U. J. Anal. Chim. Acta 2011, 706, 8.

(38) Larmour, I. A.; Graham, D. Analyst 2011, 136, 3831.

(39) Anker, J. N.; Hall, W. P.; Lyandres, O.; Shah, N. C.; Zhao, J.; Van Duyne, R. P. Nat. Mater. 2008, 7, 442.

(40) Grieshaber, D.; MacKenzie, R.; Vörös, J.; Reimhult, E. Sensors 2008, 8, 1400 .

(41) Bañuls, M. J.; Puchades, R.; Maquieira, Á. Anal. Chim. Acta 2013, 777, 1.

(42) Estevez, M. C.; Alvarez, M.; Lechuga, L. M. Laser Photonics Rev. 2012, 6, 463.

(43) Onclin, S.; Ravoo, B. J.; Reinhoudt, D. N. Angew. Chem. Int. Ed. 2005, 44, 6282.

(44) Pumera, M.; Ambrosi, A.; Bonanni, A.; Chng, E. L. K.; Poh, H. L. Trends Anal. Chem. 2010, 29, 954.

(45) Kuila, T.; Bose, S.; Khanra, P.; Mishra, A. K.; Kim, N. H.; Lee, J. H. Biosens. Bioelectron. 2011, 26, 4637.

(46) Schuler, M.; Trentin, D.; Textor, M.; Tosatti, S. G. P. Nanomedicine 2006, 1, 449.

(47) Shetti, N. P.; Bukkitgar, S. D.; Reddy, K. R.; Reddy, C. V.; Aminabhavi, T. M. Colloids Surf., B 2019, 178, 385.
(48) Jani, A. M. M.; Losic, D.; Voelcker, N. H. Prog. Mater. Sci. 2013, 58 , 636.

(49) Takmakov, P.; Vlassiouk, I.; Smirnov, S. Analyst 2006, 131, 1248.

(50) Aydin, E. B.; Sezgintürk, M. K. Trends Anal. Chem. 2017, 97, 309.

(51) Khan, M. Z. H. Cogent Eng. 2016, 3, 1.

(52) Colin, D. B.; Hans, A. B.; George, M. W. Langmuir 1989, 5, 723.

(53) Bain, C. D.; Whitesides, G. M. J. Am. Chem. Soc. 1989, 111, 7155.

(54) Walczak, M. M.; Chung, C.; Stole, S. M.; Widrig, C. A.; Porter, M. D. J. Am. Chem. Soc. 1991, 113, 2370.

(55) Fenter, P.; Eisenberger, P.; Li, J.; Camillone, N.; Bernasek, S.; Scoles, G.; Ramanarayanan, T. A.; Liang, K. S. Langmuir 1991, 7, 2013.

(56) Sagiv, J. J. Am. Chem. Soc. 1980, 102, 92.

(57) Netzer, L.; Sagiv, J. J. Am. Chem. Soc. 1983, 105, 674.

(58) Chaki, N. K.; Vijayamohanan, K. Biosens. Bioelectron. 2002, $17,1$.

(59) Flink, S.; Van Veggel, F. C. J. M.; Reinhoudt, D. N. Adv. Mater. 2000, 12, 1315.

(60) Herrwerth, S.; Eck, W.; Reinhardt, S.; Grunze, M. J. Am. Chem. Soc. 2003, 125, 9359.

(61) Samanta, D.; Sarkar, A. Chem. Soc. Rev. 2011, 40, 2567.

(62) Sassolas, A.; Leca-Bouvier, B. D.; Blum, L. J. Chem. Rev. 2008, $108,109$.

(63) Luderer, F.; Walschus, U. Top. Curr. Chem. 2005, 260, 37.

(64) Frasconi, M.; Mazzei, F.; Ferri, T. Anal. Bioanal. Chem. 2010, 398, 1545.

(65) Peluso, P.; Wilson, D. S.; Do, D.; Tran, H.; Venkatasubbaiah, M.; Quincy, D.; Heidecker, B.; Poindexter, K.; Tolani, N.; Phelan, M.; Witte, K.; Jung, L. S.; Wagner, P.; Nock, S. Anal. Biochem. 2003, $312,113$.

(66) Hao, C.; Yan, F.; Ding, L.; Xue, Y.; Ju, H. Electrochem. Commun. 2007, 9, 1359.

(67) Jing, G.; Perry, S. F.; Tatic-Lucic, S. Biomed. Microdevices 2010, 12, 935.

(68) Houseman, B. T.; Gawalt, E. S.; Mrksich, M. Langmuir 2003, 19, 1522.

(69) Knichel, M.; Heiduschka, P.; Beck, W.; Jung, G.; Göpel, W. Sens. Actuators B Chem. 1995, 28, 85.

(70) Arroyo-Currás, N.; Somerson, J.; Vieira, P. A.; Ploense, K. L.; Kippin, T. E.; Plaxco, K. W. Proc. Natl. Acad. Sci. U.S.A. 2017, 114, 645.

(71) Zhang, X.; Yadavalli, V. K. Biosens. Bioelectron. 2011, 26, 3142.

(72) Tang, Z.; Wang, Y.; Podsiadlo, P.; Kotov, N. A. Adv. Mater. 2006, 18, 3203.

(73) Park, J. W.; Kim, H.; Han, M. Chem. Soc. Rev. 2010, 39, 2935.

(74) Dobrynin, A. V.; Deshkovski, A.; Rubinstein, M. Macromolecules 2001, 34, 3421.

(75) Stuart, M. A. C.; Fleer, G. J.; Lyklema, J.; Norde, W.; Scheutjens, J. M. H. M. Adv. Colloid Interface Sci. 1991, 34, 477.

(76) Porus, M.; Maroni, P.; Borkovec, M. Langmuir 2012, 28, 5642.

(77) Boudou, T.; Crouzier, T.; Ren, K.; Blin, G.; Picart, C. Adv. Mater. 2010, 22, 441.

(78) Ruiz-Taylor, L. A.; Martin, T. L.; Zaugg, F. G.; Witte, K.; Indermuhle, P.; Nock, S.; Wagner, P. Proc. Natl. Acad. Sci. U.S. A. 2001, 98, 852.

(79) Marmisollé, W. A.; Irigoyen, J.; Gregurec, D.; Moya, S.; Azzaroni, O. Adv. Funct. Mater. 2015, 25, 4144.

(80) Decher, G.; Lehr, B.; Lowack, K.; Lvov, Y.; Schmitt, J. Biosens. Bioelectron. 1994, 9, 677.

(81) Schanze, K. S.; Shelton, A. H. Langmuir 2009, 25, 13698.

(82) Nakano, S.; Kanzaki, T.; Nakano, M.; Miyoshi, D.; Sugimoto, N. Anal. Chem. 2011, 83, 6368. 
(83) Chakrabarti, R.; Klibanov, A. M. J. Am. Chem. Soc. 2003, 125, 12531.

(84) Herne, T. M.; Tarlov, M. J. J. Am. Chem. Soc. 1997, 119, 8916.

(85) Lee, S. W.; Lopez, J.; Saraf, R. F. Electroanalysis 2013, 25, 1557.

(86) Kim, H. S.; Jung, S. H.; Kim, S. H.; Suh, I. B.; Kim, W. J.; Jung, J. W.; Yuk, J. S.; Kim, Y. M.; Ha, K. S. Proteomics 2006, 6, 6426.

(87) Purdey, M. S.; Schartner, E. P.; Sutton-McDowall, M. L.; Ritter, L. J.; Thompson, J. G.; Monro, T. M.; Abell, A. D. Proc. SPIE Int. Soc. Opt. Eng. 2015, 9506, 950614; doi: 10.1117/12.2178680.

(88) De Paul, S. M.; Falconnet, D.; Pasche, S.; Textor, M.; Abel, A. P.; Kauffmann, E.; Liedtke, R.; Ehrat, M. Anal. Chem. 2005, 77, 5831.

(89) Movilli, J.; Rozzi, A.; Ricciardi, R.; Corradini, R.; Huskens, J. Bioconjugate Chem. 2018, 29, 4110.

(90) Duan, X.; Mu, L.; Sawtelle, S. D.; Rajan, N. K.; Han, Z.; Wang, Y.; Qu, H.; Reed, M. A. Adv. Funct. Mater. 2015, 25, 2279.

(91) Sagnella, S.; Kligman, F.; Marchant, R. E.; Kottke-Marchant, K.J. Biomed. Mater. Res. 2003, 67A, 689.

(92) Stuart, M. A. C.; Hoogendam, C. W.; de Keizer, A. J. Phys.: Condens. Matter 1997, 9, 7767.

(93) Von Goeler, F.; Muthukumar, M. J. Chem. Phys. 1994, 100, 7796.

(94) Popa, I.; Cahill, B. P.; Maroni, P.; Papastavrou, G.; Borkovec, M. J. Colloid Interface Sci. 2007, 309, 28.

(95) Stuart, M. A. C. Polyelectrolytes on solid surfaces. In: Short and Long Chains at Interfaces. Daillant, J.; Guenoun, P.; Marques, C.; Muller, P.; Van, J. T. T., eds.; Atlantica-Seguier: Gif-sur-Yvette, France, 1995, 3-12.

(96) Van de Steeg, H. G. M.; Stuart, M. A. C.; de Keizer, A.; Bijsterbosch, B. H. Langmuir 1992, 8, 2538.

(97) Hoogeveen, N. G.; Stuart, M. A. C.; Fleer, G. J. J. Colloid Interface Sci. 1996, 182, 133.

(98) Rojas, O. J.; Claesson, P. M.; Muller, D.; Neuman, R. D. J. Colloid Interface Sci. 1998, 205, 77.

(99) Joanny, J. F. Eur. Phys. J. B 1999, 9, 117.

(100) Böhmer, M. R.; Evers, O. A.; Scheutjens, J. M. M. Macromolecules 1990, 23, 2288.

(101) Blaakmeer, J.; Böhmer, M. R.; Stuart, M. A. C.; Fleer, G. J. Macromolecules 1990, 23, 2301.

(102) Lappan, U.; Buchhammer, H. M.; Lunkwitz, K. Polymer 1999, $40,4087$.

(103) Choi, J.; Rubner, M. F. Macromolecules 2005, 38, 116.

(104) Kenausis, G. L.; Vörös, J.; Elbert, D. L.; Huang, N.; Hofer, R.; RuizTaylor, L.; Textor, M.; Hubbell, J. A.; Spencer, N. D. J. Phys. Chem. B 2000, 104, 3298.

(105) Choi, J. H.; Kim, S. O.; Linardy, E.; Dreaden, E. C.; Zhdanov, V. P.; Hammond, P. T.; Cho, N. J. J. Phys. Chem. B 2015, 119, 10554.

(106) Greene, G.; Yao, G.; Tannenbaum, R. Langmuir 2004, 20, 2739.

(107) Lutkenhaus, J. L.; McEnnis, K.; Hammond, P. T. Macromolecules 2008, 41, 6047.

(108) Maheshwari, N.; Kottantharayil, A.; Kumar, M.; Mukherji, S. Appl. Surf. Sci. 2010, 257, 451.

(109) Laib, S.; MacCraith, B. D. Anal. Chem. 2007, 79, 6264.

(110) Barrantes, A.; Santos, O.; Sotres, J.; Arnebrant, T. J. Colloid Interface Sci. 2012, 388, 191.

(111) Dertinger, S. K. W.; Jiang, X.; Li, Z.; Murthy, V. N.; Whitesides, G. M. Proc. Natl. Acad. Sci. U.S.A. 2002, 99, 12542.

(112) Daikuzono, C. M.; Dantas, C. A. R.; Volpati, D.; Constantino, C. J. L.; Piazzetta, M. H. O.; Gobbi, A. L.; Taylor, D. M.; Oliveira, O. N.; Riul, A. Sens. Actuators B Chem. 2015, 207, 1129.

(113) Hsieh, M. C.; Farris, R. J.; McCarthy, T. J. Macromolecules 1997, 30,8453 .
(114) Malyar, I. V.; Gorin, D. A.; Santer, S.; Stetsyura, S. V. Langmuir 2013, 29, 16058.

(115) Kim, Y. K.; Han, S. W.; Min, D. H. ACS Appl. Mater. Interfaces 2012, 4, 6545.

(116) Barten, D.; Kleijn, J. M.; Stuart, M. A. C. Phys. Chem. Chem. Phys. 2003, 5, 4258.

(117) Zavgorodnya, O.; Serpe, M. J. Colloid. Polym. Sci. 2011, 289, 591.

(118) Kumar, G.; Wang, Y. C.; Co, C.; Ho, C. C. Langmuir 2003, 19 , 10550.

(119) Hoogeveen, N. G.; Stuart, M. A. C.; Fleer, G. J. J. Colloid Interface Sci. 1996, 182, 146.

(120) Tan, Q.; Ji, J.; Barbosa, M. A.; Fonseca, C.; Shen, J. Biomaterials 2003, 24, 4699.

(121) Vermöhlen, K.; Lewandowski, H.; Narres, H. D.; Schwuger, M. J. Colloids Surf. A 2000, 163, 45.

(122) Szilagyi, I.; Trefalt, G.; Tiraferri, A.; Maroni, P.; Borkovec, M. Soft Matter 2014, 10, 2479.

(123) Pereira, S. O.; Trindade, T.; Barros-Timmons, A. Gold Bull. 2015, $48,3$.

(124) Pham, T. D.; Do, T. U.; Pham, T. T.; Nguyen, T. A. H.; Nguyen, T. K. T.; Vu, N. D.; Le, T. S.; Vu, C. M.; Kobayashi, M. Colloid Polym. Sci. 2019, 297, 13.

(125) Dange, C.; Phan, T. N. T.; André, V.; Rieger, J.; Persello, J.; Foissy, A. J. Colloid Interface Sci. 2007, 315, 107.

(126) Jiang, X.; Ortiz, C.; Hammond, P. T. Langmuir 2002, 18, 1131.

(127) Moon, J. M.; Wei, A. J. Phys. Chem. B 2005, 109, 23336.

(128) Xu, D.; Gu, J.; Wang, W.; Yu, X.; Xi, K.; Jia, X. Nanotechnology 2010, 21, 375101.

(129) Li, P.; Huang, T.; Yang, J.; Wei, S.; Cai, C.; Chen, Y.; Lee, J. M. ChemPlusChem 2015, 80, 1148.

(130) Yamanlar, S.; Sant, S.; Boudou, T.; Picart, C.; Khademhosseini, A. Biomaterials 2011, 32, 5590.

(131) Danilowicz, C.; Cortón, E.; Battaglini, F. J. Electroanal. Chem. 1998, 445, 89 .

(132) Forster, R. J.; Vos, J. G. Langmuir 1994, 10, 4330.

(133) Yang, W.; Zhou, H.; Sun, C. Macromol. Rapid Commun. 2007, 28, 265.

(134) Cortez, M. L.; González, G. A.; Battaglini, F. Electroanalysis 2011 , 23, 156.

(135) Qiu, J. D.; Liang, R. P.; Wang, R.; Fan, L. X.; Chen, Y. W.; Xia, X. H. Biosens. Bioelectron. 2009, 25, 852.

(136) Taira, S.; Yokoyama, K. Anal. Sci. 2004, 20, 267.

(137) Taira, S.; Morita, Y.; Tamiya, E.; Yokoyama, K. Anal. Sci. 2003, 19, 177.

(138) Mokhtari, H.; Pelton, R.; Jin, L.J. Colloid Interface Sci. 2014, 413, 86.

(139) Quirk, R. A.; Chan, W. C.; Davies, M. C.; Tendler, S. J. B.; Shakesheff, K. M. Biomaterials 2001, 22, 865.

(140) Ruoslahti, E. Annu. Rev. Cell Dev. Biol. 1996, 12, 697.

(141) van der Mee, L.; Chow, E. S. Y.; de Smet, L. C. P. M.; de Puit, M.; Sudhölter, E. J. R.; Jager, W. F. Anal. Methods 2015, 7, 10121.

(142) Tauhardt, L.; Kempe, K.; Gottschaldt, M.; Schubert, U. S. Chem. Soc. Rev. 2013, 42, 7998.

(143) Zhang, S.; Geryak, R.; Geldmeier, J.; Kim, S.; Tsukruk, V. V. Chem. Rev. 2017, 117, 12942.

(144) Brink, C.; Österberg, E.; Holmberg, K.; Tiberg, F. Colloids Surf. 1992, 66, 149.

(145) Sawhney, A. S.; Hubbell, J. A. Biomaterials 1992, 13, 863.

(146) Lee, S.; Spencer, N. D. Langmuir 2008, 24, 9479.

(147) Elbert, D. L.; Hubbell, J. A. J. Biomed. Mater. Res. 1998, 42, 55.

(148) Pasche, S.; Vörös, J.; Griesser, H. J.; Spencer, N. D.; Textor, M. J. Phys. Chem. B 2005, 109, 17545. 
(149) Michel, R.; Pasche, S.; Textor, M.; Castner, D. G. Langmuir 2005, $21,12327$.

(150) Lee, S.; Vörös, J. Langmuir 2005, 21, 11957.

(151) Konradi, R.; Pidhatika, B.; Mühlebach, A.; Textor, M. Langmuir 2008, 24, 613.

(152) Pidhatika, B.; Möller, J.; Vogel, V.; Konradi, R. Chimia 2008, 62, 264.

(153) Morgese, G.; Verbraeken, B.; Ramakrishna, S. N.; Gombert, Y.; Cavalli, E.; Rosenboom, J. G.; Zenobi-Wong, M.; Spencer, N. D.; Hoogenboom, R.; Benetti, E. M. Angew. Chem. Int. Ed. 2018, 57, 11667.

(154) Löfås, S. Pure Appl. Chem. 1995, 67, 829.

(155) Holland, N. B.; Qiu, Y.; Ruegsegger, M.; Marchant, R. E. Nature 1998, 392, 799.

(156) Perrino, C.; Lee, S.; Choi, S. W.; Maruyama, A.; Spencer, N. D. Langmuir 2008, 24, 8850.

(157) Schlapak, R.; Armitage, D.; Saucedo-Zeni, N.; Chrzanowski, W.; Hohage, M.; Caruana, D.; Howorka, S. Soft Matter 2009, 5, 613.

(158) VandeVondele, S.; Vörös, J.; Hubbell, J. A. Biotechnol. Bioeng. 2003, 82, 784.

(159) Movilli, J.; Di Iorio, D.; Rozzi, A.; Hiltunen, J.; Corradini, R.; Huskens, J. ACS Appl. Polym. Mater. 2019, 1, 3165.

(160) Zhen, G.; Zurcher, S.; Falconnet, D.; Xu, F.; Kuennemann, E.; Textor, M. NTA-Functionalized Poly(L-Lysine)-g-Poly(Ethylene Glycol): A Polymeric Interface for Binding and Studying 6 HisTagged Proteins. 2005 IEEE Eng. Med. Biol. 27th Annu. Conf. 2005, 1036-1038.

(161) Valderrey, V.; Wiemann, M.; Jonkheijm, P.; Hecht, S.; Huskens, J. ChemPlusChem 2019, 84, 1324.

(162) Picart, C.; Elkaim, R.; Richert, L.; Audoin, F.; Arntz, Y.; Cardoso, M. D. S.; Schaaf, P.; Voegel, J. C.; Frisch, B. Adv. Funct. Mater. 2005, 15, 83.

(163) Huang, N.; Vörös, J.; De Paul, S. M.; Textor, M.; Spencer, N. D. Langmuir 2002, 18, 220.

(164) Bellassai, N.; Marti, A.; Spoto, G.; Huskens, J. J. Mater. Chem. B 2018, 6, 7662.

(165) Saxer, S.; Portmann, C.; Tosatti, S.; Gademann, K.; Zürcher, S.; Textor, M. Macromolecules 2010, 43, 1050.

(166) Feuz, L.; Leermakers, F. A. M.; Textor, M.; Borisov, O. Langmuir 2008, 24, 7232.

(167) Pasche, S.; De Paul, S. M.; Vörös, J.; Spencer, N. D.; Textor, M. Langmuir 2003, 19, 9216.

(168) Vörös, J.; Ramsden, J. J.; Csúcs, G.; Szendrö, I.; De Paul, S. M.; Textor, M.; Spencer, N. D. Biomaterials 2002, 23, 3699.

(169) Zhen, G.; Falconnet, D.; Kuennemann, E.; Vörös, J.; Spencer, N. D.; Textor, M.; Zürcher, S. Adv. Funct. Mater. 2006, 16, 243.

(170) Fernandez-Megia, E.; Novoa-Carballal, R.; Quiñoá, E.; Riguera, R. Biomacromolecules 2007, 8, 833.

(171) Städler, B.; Falconnet, D.; Pfeiffer, I.; Höök, F.; Vörös, J. Langmuir 2004, 20, 11348.

(172) Xu, B.; Wiehle, S.; Roth, J. A.; Cristiano, R. J. Gene Ther. 1998, 5, 1235.

(173) Jeong, J. H.; Kim, B. Y.; Cho, S. H.; Kim, J-D. J. Nonlinear Opt. Phys. Mater. 2004, 13, 525.

(174) Chen, Y.; Cao, W.; Zhou, J.; Pidhatika, B.; Xiong, B.; Huang, L.; Tian, Q.; Shu, Y.; Wen, W.; Hsing, I. M.; Wu, H. ACS Appl. Mater. Interfaces 2015, 7, 2919.

(175) Schönhoff, M. J. Phys.: Condens. Matter 2003, 15, 1781.

(176) Monge, C.; Almodóvar, J.; Boudou, T.; Picart, C. Adv. Healthc. Mater. 2015, 4, 811.

(177) Decher, G.; Hong,J. D.; Schmitt,J. Macromol. Symp. 1991, 327, 321.
(178) Decher, G.; Hong, J. D. Ber. Bunsenges. Phys. Chem. 1991, 11, 1430.

(179) Decher, G.; Hong, J. D.; Schmitt, J. Thin Solid Films 1992, 210$211,831$.

(180) Lee, H.; Lee, Y.; Statz, A. R.; Rho, J.; Park, T. G.; Messersmith, P. B. Adv. Mater. 2008, 20, 1619.

(181) Jang, Y.; Park, S.; Char, K. Korean J. Chem. Eng. 2011, 28, 1149.

(182) Schlenoff, J. B.; Li, M. Ber. Bunsenges. Phys. Chem. 1996, 100, 943.

(183) Decher, G. Science 1997, 277, 1232.

(184) Ghostine, R. A.; Jisr, R. M.; Lehaf, A.; Schlenoff, J. B. Langmuir 2013, 29, 11742.

(185) McAloney, R. A.; Sinyor, M.; Dudnik, V.; Goh, M. C. Langmuir 2001, 17, 6655.

(186) Gong, X. Phys. Chem. Chem. Phys. 2013, 15, 10459.

(187) Dubas, S. T.; Schlenoff, J. B. Langmuir 2001, 17, 7725.

(188) Lavalle, P.; Gergely, C.; Cuisinier, F. J. G.; Decher, G.; Schaaf, P.; Voegel, J. C.; Picart, C. Macromolecules 2002, 35, 4458.

(189) Menchaca, J. L.; Jachimska, B.; Cuisinier, F.; Pérez, E. Colloids Surf. A 2003, 222, 185.

(190) Notley, S. M.; Eriksson, M.; Wågberg, L. J. Colloid Interface Sci. 2005, 292, 29.

(191) Schönhoff, M.; Ball, V.; Bausch, A. R.; Dejugnat, C.; Delorme, N.; Glinel, K.; Klitzing, Rv.; Steitz, R. Colloids Surf. A 2007, 303, 14.

(192) Richardson, J. J.; Cui, J.; Björnmalm, M.; Braunger, J. A.; Ejima, H.; Caruso, F. Chem. Rev. 2016, 116, 14828.

(193) Borges, J.; Mano, J. F. Chem. Rev. 2014, 114, 8883.

(194) Gentile, P.; Carmagnola, I.; Nardo, T.; Chiono, V. Nanotechnology 2015, 26, 422001.

(195) Xu, G.; Pranantyo, D.; Xu, L.; Neoh, K. G.; Kang, E. T.; Teo, S. L. M. Ind. Eng. Chem. Res. 2016, 55, 10906.

(196) Wilson, J. T.; Cui, W.; Chaikof, E. L. Nano Lett. 2008, 8, 1940.

(197) Mariani, S.; Robbiano, V.; Strambini, L. M.; Debrassi, A.; Egri, G.; Dähne, L.; Barillaro, G. Nat. Commun. 2018, 9, 5256.

(198) Cao, Z.; Gordiichuk, P. I.; Loos, K.; Sudhölter, E. J. R.; de Smet, L. C. P. M. Soft Matter 2016, 12, 1496.

(199) Wijeratne, S.; Liu, W.; Dong, J.; Ning, W.; Ratnayake, N. D.; Walker, K. D.; Bruening, M. L. ACS Appl. Mater. Interfaces 2016, 8, 10164.

(200) Bowden, N.; Terfort, A.; Carbeck, J.; Whitesides, G. M. Science 1997, 276, 233.

(201) Cheng, M.; Wang, Y.; Yu, L.; Su, H.; Han, W.; Lin, Z.; Li, J.; Hao, H.; Tong, C.; Li, X.; Shi, F. Adv. Funct. Mater. 2015, 25, 6851.

(202) Zhou, J.; Romero, G.; Rojas, E.; Ma, L.; Moya, S.; Gao, C.J. Colloid Interface Sci. 2010, 345, 241.

(203) Xu, G.; Liu, P.; Pranantyo, D.; Neoh, K. G.; Kang, E. T. ACS Sustainable Chem. Eng. 2018, 6, 3916.

(204) Chen, D.; Wu, M.; Li, B.; Ren, K.; Cheng, Z.; Ji, J.; Li, Y.; Sun, J. Adv. Mater. 2015, 27, 5882.

(205) Müller, M.; Rieser, T.; Lunkwitz, K.; Meier-Haack, J. Macromol. Rapid Commun. 1999, 20, 607.

(206) Ishigami, T.; Amano, K.; Fujii, A.; Ohmukai, Y.; Kamio, E.; Maruyama, T.; Matsuyama, H. Separ. Purif. Technol. 2012, 99, 1.

(207) Iijima, K.; Tsuji, Y.; Kuriki, I.; Kakimoto, A.; Nikaido, Y.; Ninomiya, R.; Iyoda, T.; Fukai, F.; Hashizume, M. Colloids Surf. $B$ 2017, 160, 228.

(208) Olenych, S. G.; Moussallem, M. D.; Salloum, D. S.; Schlenoff, J. B.; Keller, T. C. S. Biomacromolecules 2005, 6, 3252.

(209) Schlenoff, J. B.; Rmaile, A. H.; Bucur, C. B. J. Am. Chem. Soc. 2008, 130, 13589.

(210) Cao, L.; Qu, Y.; Hu, C.; Wei, T.; Zhan, W.; Yu, Q.; Chen, H. Adv. Mater. Interfaces 2016, 3, 1600600. 
(211) Csúcs, G.; Michel, R.; Lussi, J. W.; Textor, M.; Danuser, G. Biomaterials 2003, 24, 1713.

(212) Kam, L.; Shain, W.; Turner, J. N.; Bizios, R. Biomaterials 2001, 22, 1049.

(213) Tang, C. S.; Dusseiller, M.; Makohliso, S.; Heuschkel, M.; Sharma, S.; Keller, B.; Vörös, J. Anal. Chem. 2006, 78, 711.

(214) Stadler, B.; Huwiler, C.; Solak, H. H.; Textor, M.; Vörös, J. Nanotechnology 2005, 2, 661.

(215) Falconnet, D.; Csúcs, G.; Grandin, H. M.; Textor, M. Biomaterials 2006, 27, 3044.

(216) Dusseiller, M. R.; Schlaepfer, D.; Koch, M.; Kroschewski, R.; Textor, M. Biomaterials 2005, 26, 5917.

(217) Michel, R.; Lussi, J. W.; Csúcs, G.; Reviakine, I.; Ketterer, B.; Danuser, G.; Hubbell, J. A.; Textor, M.; Spencer, N. D. Langmuir 2002, 18, 3281.

(218) Falconnet, D.; Pasqui, D.; Park, S.; Eckert, R.; Schift, H.; Gobrecht, J.; Barbucci, R.; Textor, M. Nano Lett. 2004, 4, 1909.

(219) Tang, C. S.; Schmutz, P.; Petronis, S.; Textor, M.; Keller, B.; Vörös, J. Biotechnol. Bioeng. 2005, 91, 285.

(220) Huwiler, C.; Halter, M.; Rezwan, K.; Falconnet, D.; Textor, M.; Vörös, J. Nanotechnology 2005, 16, 3045.

(221) Kubo, T.; Wang, X.; Tong, Q.; Yan, M. Langmuir 2011, 27, 9372.

(222) Chien, H. W.; Chang, T. Y.; Tsai, W. B. Biomaterials 2009, 30, 2209.

(223) Vacheethasanee, K.; Marchant, R. E. J. Biomed. Mater. Res. 2000, $50,302$.

(224) Textor, M.; Tosatti, S. G. P.; Wieland, M.; Brunette, D. M. Use of molecular assembly techniques for tailoring the chemical properties on smooth and rough titanium surfaces. In: BioImplant Interface: Improving Biomaterials and Tissue Reaction. Ellingsen, J. E.; Lyngstadaas, S. P., eds.; CRC Press: Boca Raton, FL, 2003, 341-366.

(225) Harris, L. G.; Tosatti, S.; Wieland, M.; Textor, M.; Richards, R. G. Biomaterials 2004, 25, 4135.

(226) Huang, N. P.; Michel, R.; Vörös, J.; Textor, M.; Hofer, R.; Rossi, A.; Elbert, D. L.; Hubbell, J. A.; Spencer, N. D. Langmuir 2001, 17, 489.

(227) Bergstrand, A.; Rahmani-Monfared, G.; Östlund, A.; Nydén, M.; Holmberg, K. J. Biomed. Mater. Res. Part A 2009, 88, 608.

(228) Tosatti, S.; Schwartz, Z.; Campbell, C.; Cochran, D. L.; VandeVondele, S.; Hubbell, J. A.; Denzer, A.; Simpson, J.; Wieland, M.; Lohmann, C. H.; Textor, M.; Boyan, B. D. J. Biomed. Mater. Res. Part A 2004, 68, 458.

(229) Marie, R.; Beech, J. P.; Vörös, J.; Tegenfeldt, J. O.; Höök, F. Langmuir 2006, 22, 10103.

(230) Menzies, D. J.; Cowie, B.; Fong, C.; Forsythe, J. S.; Gengenbach, T. R.; McLean, K. M.; Puskar, L.; Textor, M.; Thomsen, L.; Tobin, M.; Muir, B. W. Langmuir 2010, 26, 13987.

(231) Winblade, N. D.; Schmökel, H.; Baumann, M.; Hoffman, A. S.; Hubbell, J. A. J. Biomed. Mater. Res. 2002, 59, 618.

(232) Winblade, N. D.; Nikolic, I. D.; Hoffman, A. S.; Hubbell, J. A. Biomacromolecules 2000, 1, 523.

(233) Lussi, J. W.; Falconnet, D.; Hubbell, J. A.; Textor, M.; Csúcs, G. Biomaterials 2006, 27, 2534.

(234) Fink, J.; Théry, M.; Azioune, A.; Dupont, R.; Chatelain, F.; Bornens, M.; Piel, M. Lab Chip 2007, 7, 672.

(235) Dermutz, H.; Grüter, R. R.; Truong, A. M.; Demkó, L.; Vörös, J.; Zambelli, T. Langmuir 2014, 30, 7037.

(236) Anderegg, F.; Geblinger, D.; Horvath, P.; Charnley, M.; Textor, M.; Addadi, L.; Geiger, B. PLoS One 2011, 6, e28583.

(237) Franks, W.; Tosatti, S.; Heer, F.; Seif, P.; Textor, M.; Hierlemann, A. Biosens. Bioelectron. 2007, 22, 1426.
(238) Lussi, J. W.; Michel, R.; Reviakine, I.; Falconnet, D.; Goessl, A.; Csúcs, G.; Hubbell, J. A.; Textor, M. Prog. Surf. Sci. 2004, 76, 55.

(239) Ricoult, S. G.; Thompson-Steckel, G.; Correia, J. P.; Kennedy, T. E.; Juncker, D. Biomaterials 2014, 35, 727.

(240) Salánki, R.; Hős, C.; Orgovan, N.; Péter, B.; Sándor, N.; Bajtay, Z.; Erdei, A.; Horvath, R.; Szabó, B. PLoS One 2014, 9, e111450.

(241) Saravia, V.; Küpcü, S.; Nolte, M.; Huber, C.; Pum, D.; Fery, A.; Sleytr, U. B.; Toca-Herrera, J. L. J. Biotechnol. 2007, 130, 247.

(242) Pidhatika, B.; Möller, J.; Benetti, E. M.; Konradi, R.; Rakhmatullina, E.; Mühlebach, A.; Zimmermann, R.; Werner, C.; Vogel, V.; Textor, M. Biomaterials 2010, 31, 9462.

(243) Cao, B.; Li, L.; Wu, H.; Tang, Q.; Sun, B.; Dong, H.; Zhe, J.; Cheng, G. Chem. Commun. 2014, 50, 3234.

(244) Cao, B.; Tang, Q.; Li, L.; Humble, J.; Wu, H.; Liu, L.; Cheng, G. Adv. Healthc. Mater. 2013, 2, 1096.

(245) Brovko, L. Y.; Meyer, A.; Tiwana, A. S.; Chen, W.; Liu, H.; Filipe, C. D. M.; Griffiths, M. W. J. Food Prot. 2009, 72, 1020.

(246) Vaterrodt, A.; Thallinger, B.; Daumann, K.; Koch, D.; Guebitz, G. M.; Ulbricht, M. Langmuir 2016, 32, 1347.

(247) Cheng, S.; Craig, W. S.; Mullen, D.; Tschopp, J. F.; Dixon, D.; Pierschbacher, M. D. J. Med. Chem. 1994, 37, 1.

(248) Schaffner, P.; Dard, M. M. Cell. Mol. Life Sci. 2003, 60, 119.

(249) Peter, B.; Farkas, E.; Forgacs, E.; Saftics, A.; Kovacs, B.; Kurunczi, S.; Szekacs, I.; Csampai, A.; Bosze, S.; Horvath, R. Sci. Rep. 2017, 7, 42220.

(250) Maddikeri, R. R.; Tosatti, S.; Schuler, M.; Chessari, S.; Textor, M.; Richards, R. G.; Harris, L. G. J. Biomed. Mater. Res. Part A 2008, $84,425$.

(251) Tosatti, S.; De Paul, S. M.; Askendal, A.; VandeVondele, S.; Hubbell, J. A.; Tengvall, P.; Textor, M. Biomaterials 2003, 24, 4949.

(252) Schuler, M.; Owen, G. R.; Hamilton, D. W.; de Wild, M.; Textor, M.; Brunette, D. M.; Tosatti, S. G. P. Biomaterials 2006, 27, 4003.

(253) Falconnet, D.; Koenig, A.; Assi, F.; Textor, M. Adv. Funct. Mater. 2004, 14, 749.

(254) Pitt, W. G.; Morris, R. N.; Mason, M. L.; Hall, M. W.; Luo, Y.; Prestwich, G. D. J. Biomed. Mater. Res. Part A 2004, 68, 95.

(255) Sagnella, S. M.; Kligman, F.; Anderson, E. H.; King, J. E.; Murugesan, G.; Marchant, R. E.; Kottke-Marchant, K. Biomaterials 2004, 25, 1249.

(256) Larsen, C. C.; Kligman, F.; Kottke-Marchant, K.; Marchant, R. E. Biomaterials 2006, 27, 4846.

(257) Thid, D.; Holm, K.; Eriksson, P. S.; Ekeroth, J.; Kasemo, B.; Gold, J. J. Biomed. Mater. Res. Part A 2008, 84, 940.

(258) Thid, D.; Bally, M.; Holm, K.; Chessari, S.; Tosatti, S.; Textor, M.; Gold, J. Langmuir 2007, 23, 11693.

(259) Tsai, W. B.; Chen, R. P. Y.; Wei, K. L.; Chen, Y. R.; Liao, T. Y.; Liu, H. L.; Lai, J. Y. Acta Biomater. 2009, 5, 3467.

(260) Tsai, W. B.; Chen, R. P. Y.; Wei, K. L.; Tan, S. F.; Lai, J. Y.J. Biomater. Sci., Polym. Ed. 2010, 21, 377.

(261) Cui, H.; Wang, Y.; Cui, L.; Zhang, P.; Wang, X.; Wei, Y.; Chen, X. Biomacromolecules 2014, 15, 3146.

(262) Guillaume-Gentil, O.; Akiyama, Y.; Schuler, M.; Tang, C.; Textor, M.; Yamato, M.; Okano, T.; Vörös, J. Adv. Mater. 2008, 20, 560.

(263) Duan, H.; Nie, S. J. Am. Chem. Soc. 2007, 129, 3333.

(264) Wattendorf, U.; Kreft, O.; Textor, M.; Sukhorukov, G. B.; Merkle, H. P. Biomacromolecules 2008, 9, 100.

(265) Wattendorf, U.; Koch, M. C.; Walter, E.; Vörös, J.; Textor, M.; Merkle, H. P. Biointerphases 2006, 1, 123.

(266) Faraasen, S.; Vörös, J.; Csúcs, G.; Textor, M.; Merkle, H. P.; Walter, E. Pharm. Res. 2003, 20, 237. 
(267) Müller, M.; Vörös, J.; Csúcs, G.; Walter, E.; Danuser, G.; Merkle, H. P.; Spencer, N. D.; Textor, M. J. Biomed. Mater. Res. Part A 2003, 66, 55.

(268) Torrecilla, D.; Lozano, M. V.; Lallana, E.; Neissa, J. I.; NovoaCarballal, R.; Vidal, A.; Fernandez-Megia, E.; Torres, D.; Riguera, R.; Alonso, M. J.; Dominguez, F. Eur. J. Pharm. Biopharm. 2013. 83, 330.

(269) Afar, D. E.; Bhaskar, V.; Ibsen, E.; Breinberg, D.; Henshall, S. M.; Kench, J. G.; Drobnjak, M.; Powers, R.; Wong, M.; Evangelista, F.; O'Hara, C.; Powers, D.; DuBridge, R. B.; Caras, I.; Winter, R.; Anderson, T.; Solvason, N.; Stricker, P. D.; Cordon-Cardo, C.; Scher, H. I.; Grygiel, J. J.; Sutherlan, R. L.; Murray, R.; Ramakrishan, V.; Law, D. A. Mol. Cancer Ther. 2004, 3, 921.

(270) Zhao, X. Y.; Schneider, D.; Biroc, S. L.; Parry, R.; Alicke, B.; Toy, P.; Xuan, J. A.; Sakamoto, C.; Wada, K.; Schulze, M.; MüllerTiemann, B.; Parry, G.; Dinter, H. Cancer Res. 2005, 65, 2846.

(271) Raichur, A. M.; Vörös, J.; Textor, M.; Fery, A. Biomacromolecules 2006, 7, 2331.

(272) Heuberger, R.; Sukhorukov, G.; Vörös, J.; Textor, M.; Möhwald, H. Adv. Funct. Mater. 2005, 15, 357.

(273) Xie, X.; Möller, J.; Konradi, R.; Kisielow, M.; Franco-Obregón, A.; Nyfeler, E.; Mühlebach, A.; Chabria, M.; Textor, M.; Lu, Z.; Reimhult, E. Biomaterials 2011, 32, 4347.

(274) Isquith, A. J.; Abbott, E. A.; Walters, P. A. Appl. Microbiol. 1972, $24,859$.

(275) Zhen, G.; Eggli, V.; Vörös, J.; Zammaretti, P.; Textor, M.; Glockshuber, R.; Kuennemann, E. Langmuir 2004, 20, 10464.

(276) Vasdekis, A. E.; O'Neil, C. P.; Hubbell, J. A.; Psaltis, D. Biomacromolecules 2010, 11, 827.

(277) Morgenthaler, S.; Zink, C.; Städler, B.; Vörös, J.; Lee, S.; Spencer, N. D.; Tosatti, S. G. P. Biointerphases 2006, 1, 156

(278) Sjövall, P.; Agnarsson, B.; Carlred, L.; Gunnarsson, A.; Höök, F. Surf. Interface Anal. 2014, 46, 707.

(279) Jajcevic, K.; Chami, M.; Sugihara, K. Small 2016, 12, 4830.

(280) Grieshaber, D.; Reimhult, E.; Vörös, J. Enzymatic biosensors towards a multiplexed electronic detection system for early cancer diagnostics. In: Proceedings of the 2nd IEEE International Conference on Nano/Micro Engineered and Molecular Systems. 2007, 402-405.

(281) Städler, B.; Bally, M.; Grieshaber, D.; Vörös, J.; Brisson, A.; Grandin, H. M. Biointerphases 2006, 1, 142.

(282) Dusseiller, M. R.; Niederberger, B.; Städler, B.; Falconnet, D.; Textor, M.; Vörös, J. Lab Chip 2005, 5, 1387.

(283) Städler, B.; Solak, H. H.; Frerker, S.; Bonroy, K.; Frederix, F.; Vörös, J.; Grandin, H. M. Nanotechnology 2007, 18, 155306.
(284) Städler, B.; Solak, H.; Frerker, S.; Grandin, M.; Vörös, J. Nanopatterning with extreme ultraviolet interference lithography for nanoelectronics and biotechnology. In: Proceedings of the 2nd IEEE International Conference on Nano/Micro Engineered and Molecular Systems. 2007, 406-410.

(285) Jeong, J. H.; Kim, B. Y.; Lee, S. J.; Kim, J. D. Chem. Phys. Lett. 2006, 421, 373.

(286) Harmsma, P. J.; Jager, W. F.; Bosma, D.; Ullien, D.; Sudhölter, E. J. R.; de Smet, L. C. P. M.; Abdulla, S. M. C.; de Boer, B. M. Opt. Express 2014, 22, 16585.

(287) Wilson, J. T.; Chaikof, E. L.; Krishnamurthy, V. R.; Cui, W.; Qu, Z. J. Am. Chem. Soc. 2010, 132, 2845.

(288) Lepoitevin, M.; Jamilloux, B.; Bechelany, M.; Balanzat, E.; Janot, J. M.; Balme, S. RSC Adv. 2016, 6, 32228.

(289) Kim, K.; Lee, Y. M.; Lee, H. B.; Shin, K. S. Biosens. Bioelectron. 2009, 24, 3615.

(290) Burg, T. P.; Godin, M.; Knudsen, S. M.; Shen, W.; Carlson, G.; Foster, J. S.; Babcock, K.; Manalis, S. R. Nature 2007, 446, 1066.

(291) Dahlin, A. B.; Jönsson, P.; Jonsson, M. P.; Schmid, E.; Zhou, Y.; Höök, F. ACS Nano 2008, 2, 2174.

(292) Wen, X.; He, H.; Lee, L. J. J. Immunol. Methods 2009, 350, 97.

(293) Davila, J.; Toulemon, D.; Garnier, T.; Garnier, A.; Senger, B.; Voegel, J. C.; Mésini, P. J.; Schaaf, P.; Boulmedais, F.; Jierry, L. Langmuir 2013, 29, 7488.

(294) Han, Z.; Wang, Y.; Duan, X. Anal. Chim. Acta 2017, 964, 170.

(295) Liu, W.; Pan, S.; Zhang, H.; Tang, Z.; Liang, J.; Wang, Y.; Zhang, M.; Hu, X.; Pang, W.; Duan, X. ACS Cent. Sci. 2018, 4, 899.

(296) Boyer, C.; Bousquet, A.; Rondolo, J.; Whittaker, M. R.; Stenzel, M. H.; Davis, T. P. Macromolecules 2010, 43, 3775.

(297) De Geest, B. G.; Jonas, A. M.; Demeester, J.; De Smedt, S. C. Langmuir 2006, 22, 5070

(298) Taira, S.; Yokoyama, K. Biotechnol. Bioeng. 2004, 88, 35.

(299) Di Iorio, D.; Marti, A.; Koeman, S.; Huskens, J. RSC Adv. 2019, 9, 35608.

(300) Regatos, D.; Sepúlveda, B.; Fariña, D.; Carrascosa, L. G.; Lechuga, L. M. Opt. Express 2011, 19, 8336.

(301) Gunnarsson, A.; Jönsson, P.; Marie, R.; Tegenfeldt, J. O.; Höök, F. Nano. Lett. 2008, 8, 183.

(302) Hsiao, Y. S.; Ho, B. C.; Yan, H. X.; Kuo, C. W.; Chueh, D. Y.; Yu, H. H.; Chen, P. J. Mater. Chem. B 2015, 3, 5103.

(303) Sugai, H.; Tomita, S.; Ishihara, S.; Kurita, R. ACS Sens. 2019, 4, 827.

(304) Tomita, S.; Ishihara, S.; Kurita, R. ACS Appl. Mater. Interfaces 2017, 9, 22970.

(305) Sugai, H.; Tomita, S.; Ishihara, S.; Kurita, R. Sens. Mater. 2019, 31, 1. 